Pacific

Journal of

Mathematics

EXISTENCE AND UNIQUENESS OF SOLUTIONS ON BOUNDED DOMAINS TO A FITZHUGH-NAGUMO TYPE ELLIPTIC SYSTEM

Carolus Reinecke and Guido Sweers

Volume $197 \quad$ No. 1

January 2001 


\title{
EXISTENCE AND UNIQUENESS OF SOLUTIONS ON BOUNDED DOMAINS TO A FITZHUGH-NAGUMO TYPE ELLIPTIC SYSTEM
}

\author{
Carolus Reinecke and Guido Sweers
}

\begin{abstract}
In this paper we prove the existence and uniqueness of the boundary layer solution to a semilinear eigenvalue problem consisting of a coupled system of two elliptic partial differential equations. Although the system is not quasimonotone, there exists a transformation to a quasimonotone system. For the transformed system we may and will use maximum (sweeping) principle arguments to derive pointwise estimates. A degree argument completes the uniqueness proof.
\end{abstract}

\section{Introduction.}

We consider the following nonlinear eigenvalue problem:

$$
\left\{\begin{aligned}
-\Delta u & =\lambda(f(u)-v) & & \text { in } \Omega, \\
-\Delta v & =\lambda(\delta u-\gamma v) & & \text { in } \Omega, \\
u & =v=0 & & \text { on } \Gamma=\partial \Omega,
\end{aligned}\right.
$$

with $\lambda, \delta, \gamma>0$ and where $\Omega \subset \mathbb{R}^{N}$ is a smooth bounded domain. As usual, a domain is an open connected set. The nonlinearity $f$ is assumed to be smooth and like a third order polynomial. We prove the existence of a curve of positive solutions $\left(u_{\lambda}, v_{\lambda}\right)$ to $\left(\mathrm{P}_{\lambda}\right)$ for $\lambda$ large enough. These solutions are shown to be, except for a boundary layer of width $O\left(\lambda^{-1 / 2}\right)$, close to $(\rho,(\delta / \gamma) \rho)$ where $\rho$ a positive zero of $f(s)-(\delta / \gamma) s$ and $f^{\prime}(\rho)<0$. The stability of these solutions as equilibria of the parabolic system

$$
\left\{\begin{aligned}
u_{t} & =\Delta u+\lambda(f(u)-v) & & \text { in } \mathbb{R}^{+} \times \Omega, \\
v_{t} & =\Delta v+\lambda(\delta u-\gamma v) & & \text { in } \mathbb{R}^{+} \times \Omega, \\
u & =v=0 & & \text { on } \mathbb{R}^{+} \times \Gamma,
\end{aligned}\right.
$$

with appropriate initial conditions is also proven. Finally it is shown that these solutions are unique in an appropriate order interval.

The question of existence of solutions to $\left(\mathrm{P}_{\lambda}\right)$ with $\lambda=1$ and with different kinds of nonlinearities was studied by Klaasen and Mitidieri [9] and De Figueiredo and Mitidieri [7], see also Rothe [21] and Lazer and McKenna $[\mathbf{1 2}]$. The fact that the second equation can be inverted to solve $v$ in terms 
of $u$ and that the problem can then be written as a single equation in $u$ was used extensively. In particular this single equation can be treated by variational techniques. Using this approach it was shown for example in [9] with $f(u)=u(u-1)(a-u), 0<a<1 / 2$ and in [7] for more general $f$ of the same type, that there exist at least two nontrivial solutions, under the assumptions that $\delta / \gamma$ is small enough and $\Omega$ contains a large enough ball. By rescaling, this implies that there exist nontrivial solutions to $\left(\mathrm{P}_{\lambda}\right)$ if $\lambda$ is large and $\delta / \gamma$ small.

Our treatment of the problem differs from the variational approach mentioned above. By imposing some natural restrictions on the parameters, which are satisfied if $\delta / \gamma$ is small, it is possible to make a transformation of $\left(\mathrm{P}_{\lambda}\right)$ and a modification of $f$ to obtain a quasimonotone system. Solutions to the quasimonotone system in a certain range correspond to solutions to the original problem. This approach was also used in [19] as well as in [14] for other systems of equations. The advantage of working with a quasimonotone system is that for such systems a comparison principle holds. From this follows the existence of solutions between an ordered pair of sub- and supersolutions. For such systems one also has an analogue of McNabb's sweeping principle, see $[\mathbf{1 5}],[\mathbf{2}],[\mathbf{4}]$ and $[\mathbf{2 2}]$. This will be a main tool in many of the proofs.

Using this quasimonotone approach we are able to give a complete qualitative description of a specific solution to $\left(\mathrm{P}_{\lambda}\right)$. This qualitative description allows us to prove uniqueness and stability results. Results in this direction were obtained by Lazer and McKenna [12] for a system with $\delta=\gamma$ and $f$ such that $f(s) / s$ is decreasing on $\mathbb{R}^{+}$. Existence and positivity of solutions were considered in $[\mathbf{9}]$ and $[\mathbf{7}]$.

If we set $\delta=0$ in $\left(\mathrm{P}_{\lambda}\right)$ then the problem reduces to the well studied scalar problem

$$
\left\{\begin{aligned}
-\Delta u & =\lambda f(u) & & \text { in } \Omega \\
u & =0 & & \text { on } \Gamma .
\end{aligned}\right.
$$

There is an extensive literature on such kind of problems. We just mention $[\mathbf{2}],[4],[\mathbf{1 3}]$ and more recently [5]. We note that our treatment of the quasimonotone system is similar to the treatment of problem $\left(S_{\lambda}\right)$ as was done in [2] and [4]. The results of the present paper were announced in [20].

The structure of the paper is as follows. In the next section the precise assumptions on the nonlinearity $f$ are stated, as well as the conditions which we impose on the parameters $\gamma$ and $\delta$. It is then shown how $\left(\mathrm{P}_{\lambda}\right)$ can be transformed to a quasimonotone system. The main results are also stated in this section. In Section 3 we prove several auxiliary results. The proofs of the main theorems are given in Section 4. In Appendix A we define our notion of sub- and supersolutions for quasimonotone systems and give some 
related results. In particular we state a version of the sweeping principle for quasimonotone systems. This principle is used repeatedly in the proofs.

\section{Assumptions and main results.}

The assumptions on $f$ are the following.

Condition A. The function $f \in C^{1,1}(\mathbb{R}), f(0) \geq 0$ and there exists $\sigma_{0}>0$ such that for every $0 \leq \sigma<\sigma_{0}$ there exist $\rho_{\sigma}^{-}<\rho_{\sigma}^{+}$with $\rho_{\sigma}^{+}>0$ such that

(1) $f\left(\rho_{\sigma}^{ \pm}\right)=\sigma \rho_{\sigma}^{ \pm}$and $f(s)>\sigma s$ for $\rho_{\sigma}^{-}<s<\rho_{\sigma}^{+}$;

(2) $f^{\prime}(s)<0$ for all $s \in\left(\rho_{\sigma_{0}}^{+}, \rho_{0}^{+}\right)$;

(3) $J_{\sigma}(\rho)>0$ on $\left(0, \rho_{\sigma}^{+}\right)$for all $0 \leq \sigma<\sigma_{0}$ where

$$
J_{\sigma}(\rho):=\int_{\rho}^{\rho_{\sigma}^{+}}(f(s)-\sigma s) d s .
$$

See Figure 1.

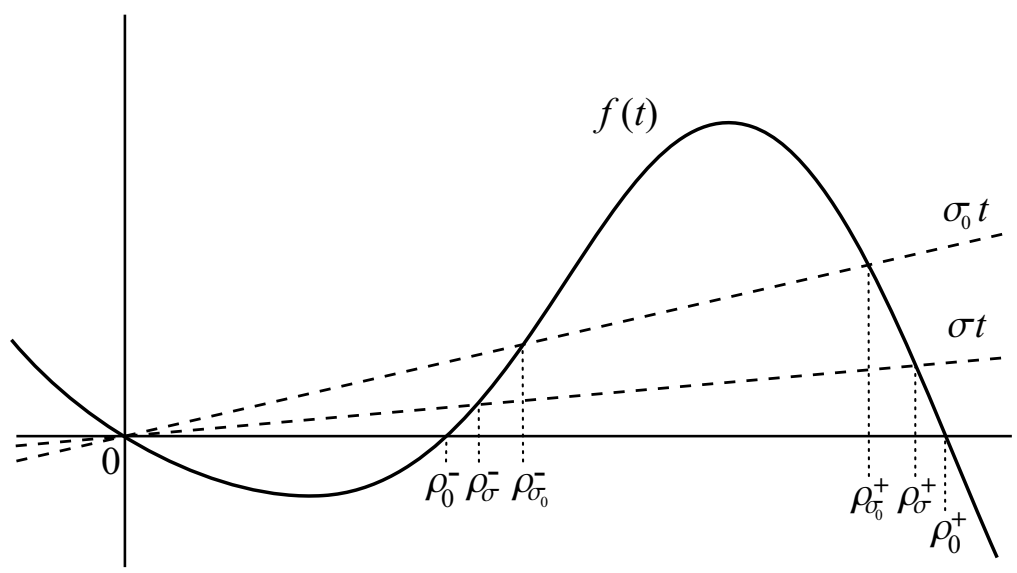

Figure 1.

Example 1. The function $f(u)=a u-u^{3}$ with $a>0$, see [12] and [7], satisfies Condition A above with $\sigma_{0}=2 a / 3$.

Example 2. Consider the function $f(u)=u(a-u)(u-1)$ with $a>0$. Condition A holds if $a<1 / 2$. In this case $\sigma_{0}=\left(2 a^{2}-5 a+2\right) / 9$. With this nonlinearity problem $\left(\mathrm{P}_{\lambda}\right)$ is an extension of the FitzHugh-Nagumo equations, see [9] and [10].

As was said in the introduction, an important step in our analysis is to transform $\left(\mathrm{P}_{\lambda}\right)$ and to modify $f$ in order to obtain a quasimonotone system. For the definition of a quasimonotone system and some results for such 
systems we refer to Appendix A. In order to transform system $\left(\mathrm{P}_{\lambda}\right)$ we need the following assumption on the parameters $\delta$ and $\gamma$ :

Condition B1. Let $M:=\max \left\{-f^{\prime}(s) ; 0 \leq s \leq \rho_{0}^{+}\right\}$and suppose that

$$
\gamma-2 \sqrt{\delta}>M
$$

We define $\beta$ and $\alpha$ by

$$
\begin{gathered}
\beta:=\frac{1}{2}(\gamma-M)-\frac{1}{2} \sqrt{(\gamma-M)^{2}-4 \delta}, \\
\alpha=\gamma-\beta .
\end{gathered}
$$

If Condition B1 holds then $\beta \in \mathbb{R}$ and $\alpha, \beta>0$. Note that $-\beta(\beta+M)=$ $\delta-\gamma \beta$ and that

$$
\vartheta:=1-\frac{\delta}{\gamma \beta}>0 .
$$

One may verify that $(u, w)$ is a solution to

$$
\left\{\begin{aligned}
-\Delta u & =\lambda(f(u)-\beta u+\beta w) & & \text { in } \Omega, \\
-\Delta w & =\lambda(f(u)+M u-\alpha w) & & \text { in } \Omega, \\
u & =w=0 & & \text { on } \Gamma,
\end{aligned}\right.
$$

if and only if $(u, \beta u-\beta w)$ is a solution to $\left(\mathrm{P}_{\lambda}\right)$.

Let $\tilde{f} \in C^{1,1}(\mathbb{R})$ be a function satisfying $\tilde{f}(s)=f(s)$ for all $s \in\left[0, \rho_{0}^{+}\right]$ with $\tilde{f}, \tilde{f}^{\prime}$ bounded on $\mathbb{R}$ and with $\tilde{f}^{\prime}(s)+M \geq 0$ for all $s \in \mathbb{R}$. If we replace $f$ in $\left(\mathrm{Q}_{\lambda}\right)$ by $\tilde{f}$ the system becomes quasimonotone. Since we are interested in solutions $(u, v)$ to $\left(\mathrm{P}_{\lambda}\right)$ with $u$ positive and $\max u<\rho_{\delta / \gamma}^{+} \leq \rho_{0}^{+}$we can assume without loss of generality the following:

Condition $\mathbf{A}^{*}$. The function $f$ satisfies Condition $\mathrm{A}$ with $f$ and $f^{\prime}$ bounded and $f^{\prime}(s)+M \geq 0$ for all $s \in \mathbb{R}$ and $f(s) \leq 0$ for $s \geq \rho_{0}^{+}$.

Another condition which we impose is:

Condition B2. The constant $\beta$ defined in (3) satisfies $\beta<\sigma_{0}$.

Under this condition one has for $\lambda$ large enough a positive nontrivial solution to the scalar problem

$$
\left\{\begin{aligned}
-\Delta u & =\lambda(f(u)-\beta u) & & \text { in } \Omega, \\
u & =0 & & \text { on } \Gamma,
\end{aligned}\right.
$$

which has its maximum in the interval $\left(\rho_{\beta}^{-}, \rho_{\beta}^{+}\right)$, see [4]. This solution will be used to obtain a nontrivial subsolution to $\left(\mathrm{Q}_{\lambda}\right)$ for $\lambda$ large enough. The definition of sub- and supersolutions is given in Appendix A. 
We make some remarks on Conditions B1 and B2. Both conditions are satisfied if $\delta / \gamma$ is small enough. More precisely, for fixed $\delta>0, \mathrm{~B} 1$ and B2 are satisfied if

$$
\gamma> \begin{cases}M+2 \sqrt{\delta} & \text { if } \quad 0 \leq \delta<\sigma_{0}^{2} \\ M+\sigma_{0}+\delta / \sigma_{0} & \text { if } \delta \geq \sigma_{0}^{2}\end{cases}
$$

In the first theorem we prove the existence of a curve of positive solutions to $\left(\mathrm{P}_{\lambda}\right)$.

Theorem 2.1 (Existence of a curve of solutions). Let $f$ satisfy Condition $\mathrm{A}$, let $\gamma, \delta$ be such that Conditions B1 and $\mathrm{B} 2$ hold and assume that $\Gamma$ is $C^{3}$. Then there exist $\lambda^{\star}>0$ and a function $\Lambda \in C^{1}\left(\left[\lambda^{\star},+\infty\right), C^{2}(\bar{\Omega}) \times C^{2}(\bar{\Omega})\right)$ such that $\left(u_{\lambda}, v_{\lambda}\right):=\Lambda(\lambda)$ is a positive solution, i.e., $u_{\lambda}, v_{\lambda} \geq 0$, to $\left(\mathrm{P}_{\lambda}\right)$ for all $\lambda \geq \lambda^{\star}$. Furthermore

(1) $\max u_{\lambda} \in\left(\rho_{\delta / \gamma}^{-}, \rho_{\delta / \gamma}^{+}\right)$and $\max v_{\lambda} \in \frac{\delta}{\gamma}\left(\rho_{\delta / \gamma}^{-}, \rho_{\delta / \gamma}^{+}\right)$;

(2) $\lim _{\lambda \rightarrow \infty} \Lambda(\lambda)=\left(\rho_{\delta / \gamma}^{+}, \frac{\delta}{\gamma} \rho_{\delta / \gamma}^{+}\right)$uniformly on compact subsets of $\Omega$.

The stability of the solutions obtained in the theorem above will be considered in the space $X:=C(\bar{\Omega}) \times C(\bar{\Omega})$. For $\lambda>\lambda^{\star}$ we define the linear operator $A_{\lambda}: D\left(A_{\lambda}\right) \subset X \rightarrow X$ by

$$
D\left(A_{\lambda}\right):=\{(u, v) \in X ;(\Delta u, \Delta v) \in X\}
$$

and

$$
A_{\lambda}\left(\begin{array}{l}
u \\
v
\end{array}\right):=\left(\begin{array}{rr}
-\Delta & 0 \\
0 & -\Delta
\end{array}\right)\left(\begin{array}{l}
u \\
v
\end{array}\right)-\lambda\left(\begin{array}{cc}
f^{\prime}\left(u_{\lambda}\right) & 1 \\
\delta & -\gamma
\end{array}\right)\left(\begin{array}{l}
u \\
v
\end{array}\right)
$$

for $(u, v) \in D\left(A_{\lambda}\right)$. Here $u_{\lambda}$ is the first component of $\Lambda(\lambda)$. In the definition of $D\left(A_{\lambda}\right), \Delta u$ and $\Delta w$ are to be understood in distributional sense.

Theorem 2.2 (Stability). Assume that the conditions of Theorem 2.1 hold and let $\lambda^{\star}$ and $\Lambda$ be as in that theorem. For every $\lambda \geq \lambda^{\star}$ the solution $\Lambda(\lambda)=\left(u_{\lambda}, v_{\lambda}\right)$ to $\left(\mathrm{P}_{\lambda}\right)$ is an exponentially stable equilibrium solution to the initial value problem (1) i.e., for every $\lambda \geq \lambda^{\star}$ there exists $\nu_{\lambda}>0$ such that the spectrum $\sigma\left(A_{\lambda}\right)$ is contained in $\left\{\nu \in \mathbb{C} ; \operatorname{Re} \nu>\nu_{\lambda}\right\}$.

Our last theorem is a result on the uniqueness, in a restricted sense, of solutions to $\left(\mathrm{P}_{\lambda}\right)$.

Theorem 2.3 (Uniqueness in order interval). Assume that the conditions of Theorem 2.1 hold and let $\lambda^{\star}$ and $\Lambda$ be as in that theorem. For every function $z \in C_{0}(\Omega)$ with $z \geq 0$ and $\max z \in\left(\rho_{\beta}^{-}, \rho_{\delta / \gamma}^{+}\right)$there exists $\lambda_{z}>\lambda^{\star}$ such that if $(u, v)$ is a solution to $\left(\mathrm{P}_{\lambda}\right)$ with $\lambda>\lambda_{z}$ and $u \in\left[z, \rho_{\delta / \gamma}^{+}\right]$then $(u, v)=\Lambda(\lambda)$. 
In general one cannot expect uniqueness of solutions. Indeed it may for example be the case that the trivial solution is a stable solution to the problem. Then there will exist a third, unstable solution in $[0, \Lambda(\lambda)]$. This is the case when $f$ is as in Example 2 and Conditions B1 and B2 hold, see $[19]$.

We end this section with a summary of the notation that will be used.

\section{Notation:}

- Let $u_{1}, u_{2} \in C(\bar{\Omega})$. We write

$$
\begin{aligned}
& u_{1} \geq u_{2} \text { if } u_{1}(x) \geq u_{2}(x) \text { for all } x \in \Omega ; \\
& u_{1} \supsetneqq u_{2} \text { if } u_{1} \geq u_{2} \text { and } u_{1} \neq u_{2} ; \\
& u_{1}>u_{2} \text { if } u_{1}(x)>u_{2}(x) \text { for all } x \in \Omega .
\end{aligned}
$$

- For $(u, w) \in C(\bar{\Omega}) \times C(\bar{\Omega})$ we shall use $(u, w)(x)=(u(x), w(x))$.

- Let $\left(u_{i}, w_{i}\right) \in C(\bar{\Omega}) \times C(\bar{\Omega}), i=1,2$. We write

$$
\begin{aligned}
& \left(u_{1}, w_{1}\right) \geq\left(u_{2}, w_{2}\right) \text { if } u_{1} \geq u_{2} \text { and } w_{1} \geq w_{2} ; \\
& \left(u_{1}, w_{1}\right) \supsetneqq\left(u_{2}, w_{2}\right) \text { if }\left(u_{1}, w_{1}\right) \geq\left(u_{2}, w_{2}\right) \text { and }\left(u_{1}, w_{1}\right) \neq\left(u_{2}, w_{2}\right) ; \\
& \left(u_{1}, w_{1}\right)>\left(u_{2}, w_{2}\right) \text { if } u_{1}>u_{2} \text { and } w_{1}>w_{2} .
\end{aligned}
$$

- If $\left(u_{1}, w_{1}\right) \geq\left(u_{2}, w_{2}\right)$ we denote by $\left[\left(u_{1}, w_{1}\right),\left(u_{2}, w_{2}\right)\right]$ the order interval

$$
\left\{(u, w) \in C(\bar{\Omega}) \times C(\bar{\Omega}) ;\left(u_{1}, w_{1}\right) \leq(u, w) \leq\left(u_{2}, w_{2}\right)\right\} .
$$

- By $\mathcal{D}^{+}(\Omega)$ we denote the set of $z \in C_{0}^{\infty}(\Omega)$ with $z \geq 0$ and $\mathcal{D}^{\prime}(\Omega)$ denotes the usual space of distributions.

- For $u_{1}, u_{2} \in C(\bar{\Omega})$ we say $-\Delta u_{1} \leq u_{2}$ in $\mathcal{D}^{\prime}(\Omega)$-sense if

$$
\int_{\Omega} u_{1}(-\Delta z) d x \leq \int_{\Omega} u_{2} z d x
$$

for all $z \in \mathcal{D}^{+}(\Omega)$.

- For a Banach space $X$ we denote the bounded linear operators from $X$ into $X$ by $\mathcal{L}(X)$.

\section{Preliminary results.}

\subsection{Estimates for positive solutions.}

Proposition 3.1. Let $B$ be the unit ball in $\mathbb{R}^{N}$. Suppose that $f$ satisfies Condition $\mathrm{A}^{*}$. Then there exists $\lambda_{B}>0$ such that the problem

$$
\left\{\begin{aligned}
-\Delta u & =\lambda_{B}(f(u)-\beta u+\beta w) & & \text { in } B, \\
-\Delta w & =\lambda_{B}(f(u)+M u-\alpha w) & & \text { in } B, \\
u & =w=0 & & \text { on } \partial B,
\end{aligned}\right.
$$

has a solution $\left(U_{B}, W_{B}\right)$ with the following properties: 
(1) $0 \leq\left(U_{B}, W_{B}\right)<\left(\rho_{\delta / \gamma}^{+}, \vartheta \rho_{\delta / \gamma}^{+}\right)$, with $\vartheta=1-\delta /(\gamma \beta)$.

(2) $U_{B}$ and $W_{B}$ are radially symmetric with

$$
U_{B}^{\prime}(0)=W_{B}^{\prime}(0)=0 \quad \text { and } \quad U_{B}^{\prime}(r), W_{B}^{\prime}(r)<0 \quad \text { on }(0,1] .
$$

(3) $\left(U_{B}(0), W_{B}(0)\right)>\left(\rho_{\delta / \gamma}^{-}, \vartheta \rho_{\delta / \gamma}^{-}\right)$and $W_{B}(0) \geq \vartheta \tau$ where $\tau:=U_{B}(0)$.

Proof. Since Condition B2 holds, $J_{\beta}(\rho)>0$ for all $0 \leq \rho<\rho_{\beta}^{+}$. This implies that for $\lambda$ large enough, say $\lambda=\lambda_{B}$, there exists a positive solution $\underline{u}$ to

$$
\left\{\begin{aligned}
-\Delta u & =\lambda(f(u)-\beta u) & & \text { in } B \\
u & =0 & & \text { on } \partial B
\end{aligned}\right.
$$

with $\max \underline{u} \in\left(\rho_{\beta}^{-}, \rho_{\beta}^{+}\right)$, see [4]. Then $(\underline{u}, 0)$ is a subsolution to (8). Since $\left(\rho_{\delta / \gamma}^{+}, \vartheta \rho_{\delta / \gamma}^{+}\right)$is a supersolution with $(\underline{u}, 0)<\left(\rho_{\delta / \gamma}^{+}, \vartheta \rho_{\delta / \gamma}^{+}\right)$there exists a solution $\left(U_{B}, W_{B}\right)$ with $\underline{u}<U_{B}<\rho_{\delta / \gamma}^{+}$and $0<W_{B}<\vartheta \rho_{\delta / \gamma}^{+}$to (8), see Proposition A.3. Using an extension due to Troy, [24], of results of Gidas, $\mathrm{Ni}$ and Nirenberg, [8], to quasimonotone systems, we have that $U_{B}$ and $W_{B}$ are radially symmetric with $U_{B}^{\prime}(0)=W_{B}^{\prime}(0)=0$ and $U_{B}^{\prime}(r), W_{B}{ }^{\prime}(r)<0$ on the interval $(0,1)$. Also $\left(-\Delta+\lambda_{B} \alpha\right) W_{B}=\lambda_{B}\left(f\left(U_{B}\right)+M U_{B}\right) \geq 0$ and by the strong maximum principle $W_{B}^{\prime}(1)<0$. Let $\tau:=U_{B}(0)$. With $V_{B}=\beta\left(U_{B}-W_{B}\right)$ it also follows from the maximum principle that

$$
\max V_{B}<(\delta / \gamma) \tau \text {. }
$$

Indeed, $\left(-\Delta+\lambda_{B} \gamma\right)\left(V_{B}-\delta \tau / \gamma\right)=\lambda_{B}\left(U_{B}-\tau\right) \leq 0$ in $B$, with $V_{B}=0$ on $\partial B$ and (9) follows. Since $V_{B}$ is also radially symmetric and decreasing, $V_{B}(0)=\beta\left(\tau-W_{B}(0)\right)<(\delta / \gamma) \tau$ and hence

$$
W_{B}(0)>(1-\delta /(\gamma \beta)) \tau=\vartheta \tau>\vartheta \rho_{\delta / \gamma}^{-} .
$$

Also $V_{B}^{\prime}(1)=\beta\left(U_{B}^{\prime}(1)-W_{B}^{\prime}(1)\right)<0$ and hence $U_{B}^{\prime}(1)<W_{B}^{\prime}(1)<0$.

Next we construct a family of subsolutions to $\left(Q_{\lambda}\right)$ using the functions $U_{B}$ and $W_{B}$. These subsolutions will be used to determine by sweeping the shape of the solutions to $\left(\mathrm{Q}_{\lambda}\right)$ in a certain order interval. We fix $z^{*} \in \Omega$ and let

$$
\lambda^{*}:=\lambda_{B} \operatorname{dist}\left(z^{*}, \Gamma\right)^{-2} .
$$

Lemma 3.2. For all $\lambda \geq \lambda^{*}$ we set

$$
Z_{\lambda}(x):=\left\{\begin{array}{lll}
\left(U_{B}, W_{B}\right)\left(\left(\lambda / \lambda_{B}\right)^{1 / 2}\left(x-z^{*}\right)\right) & \text { for } & \left|x-z^{*}\right| \leq\left(\lambda_{B} / \lambda\right)^{1 / 2} \\
0 & \text { for } & \left|x-z^{*}\right|>\left(\lambda_{B} / \lambda\right)^{1 / 2},
\end{array}\right.
$$

with $\left(U_{B}, W_{B}\right)$ as in Proposition 3.1. Then $Z_{\lambda}$ is a subsolution to $\left(\mathrm{Q}_{\lambda}\right)$ and

$$
Y:=\left(\rho_{\delta / \gamma}^{+}, \vartheta \rho_{\delta / \gamma}^{+}\right)
$$

is a supersolution to $\left(\mathrm{Q}_{\lambda}\right)$ with $Z_{\lambda}<Y$. 
Proof. It follows directly that $Y$ is a supersolution. The function $Z_{\lambda}$ is continuous and $Z_{\lambda}(x)=0$ for $x \in \Gamma$. Denote by $Z_{\lambda, i}, i=1,2$, the two components of $Z_{\lambda}$. Let $z \in \mathcal{D}^{+}(\Omega)$. Then, with $B_{\lambda}=B\left(z^{*},\left(\lambda_{B} / \lambda\right)^{1 / 2}\right)$ and $n$ denoting the outward normal, we obtain by the Green identity:

$$
\begin{aligned}
\int_{\Omega} Z_{\lambda, 1}(-\Delta z) d x & =\int_{B_{\lambda}} Z_{\lambda, 1}(-\Delta z) d x \\
& =-\int_{B_{\lambda}}\left(\Delta Z_{\lambda, 1}\right) z d x-\int_{\partial B_{\lambda}}\left(Z_{\lambda, 1} \frac{\partial z}{\partial n}-z \frac{\partial Z_{\lambda, 1}}{\partial n}\right) d S \\
& \leq \lambda \int_{\Omega}\left(f\left(Z_{\lambda, 1}\right)-\beta Z_{\lambda, 1}+\beta Z_{\lambda, 2}\right) z d x .
\end{aligned}
$$

A similar result holds for $Z_{\lambda, 2}$. Finally $\max Z_{\lambda, 1}=Z_{\lambda, 1}\left(z^{*}\right)=\tau<\rho_{\delta / \gamma}^{+}$and $\max Z_{\lambda, 2}=Z_{\lambda, 2}\left(z^{*}\right)=W_{B}(0)<\vartheta \rho_{\delta / \gamma}^{+}$.

Since $Z_{\lambda}$ is a subsolution to $\left(\mathrm{Q}_{\lambda}\right)$ and $Y$ is a supersolution to $\left(\mathrm{Q}_{\lambda}\right)$ with $Z_{\lambda}<Y$ there exists at least one solution in the order interval $\left[Z_{\lambda}, Y\right]$. For every fixed $\lambda \geq \lambda^{*}$ we define for all $y \in \Omega$ satisfying $\operatorname{dist}(y, \Gamma)>\left(\lambda_{B} / \lambda\right)^{-1 / 2}$ the functions

$$
Z_{\lambda}^{y}(x):=Z_{\lambda}\left(x+z^{*}-y\right) .
$$

Repeating the proof of Lemma 3.2 one sees that for $\lambda \geq \lambda^{*}$,

$$
\mathcal{S}_{\lambda}:=\left\{Z_{\lambda}^{y} ; y \in \Omega \text { such that } \operatorname{dist}(y, \Gamma)>\left(\lambda_{B} / \lambda\right)^{1 / 2}\right\}
$$

is a family of subsolutions. We shall use the sweeping principle with functions in $\mathcal{S}_{\lambda}$ to obtain, at least for $\lambda$ large enough, estimates of solutions to $\left(\mathrm{Q}_{\lambda}\right)$ in the order interval $\left[Z_{\lambda}, Y\right]$. In order to estimate a solution in $\left[Z_{\lambda}, Y\right]$ in all of $\Omega$ as well as on the boundary we make the following assumption on $\Gamma$ which holds if $\Gamma \in C^{3}$ :

- $\Omega$ satisfies a uniform interior sphere condition, that is, there exists $\varepsilon_{\Omega}>0$ such that $\Omega=\cup\left\{B(y, \varepsilon) ; y \in \Omega\right.$ and $\left.\operatorname{dist}(y, \Gamma)>\varepsilon_{\Omega}\right\}$.

We may suppose that $\Omega_{\varepsilon}:=\{y \in \Omega$; $\operatorname{dist}(y, \Gamma)>\varepsilon\}$ is connected for all $\varepsilon \leq \varepsilon_{\Omega}$.

Lemma 3.3. There exists $\lambda^{\times}>\lambda^{*}$ and $b>0$ such that for all $\lambda>\lambda^{\times}$we have the following estimate for every solution $(u, w) \in\left[Z_{\lambda}, Y\right]$ to $\left(\mathrm{Q}_{\lambda}\right)$ :

$$
(u(x), w(x))>\min \left\{b \lambda^{1 / 2} \operatorname{dist}(x, \Gamma), \tau\right\}(1, \vartheta),
$$

with $\vartheta=1-\delta /(\gamma \beta)$ and $\tau$ as in Proposition 3.1.

Proof. Let $\varepsilon_{\lambda}:=\left(\lambda_{B} / \lambda\right)^{1 / 2}$ and $\lambda^{\times}:=\max \left\{\lambda^{*}, \lambda_{B} \varepsilon_{\Omega}^{-2}\right\}$. Suppose that $(u, w) \in\left[Z_{\lambda}, Y\right]$ is a solution to $\left(\mathrm{Q}_{\lambda}\right)$ with $\lambda>\lambda^{\times}$. As in [4] there exists for every $y \in \Omega_{\varepsilon_{\lambda}}$ a curve in $\Omega_{\varepsilon_{\lambda}}$ connecting $y$ with $z^{*}$. Using the sweeping 
principle, Proposition A.6, it follows that $(u, w)>Z_{\lambda}^{y}$ for all $y \in \Omega_{\varepsilon_{\lambda}}$. Using $(u(x), w(x)) \geq \sup _{y \in \Omega_{\varepsilon_{\lambda}}} Z_{\lambda}^{y}(x)$ one finds (13).

The next lemma improves the estimate we found in the previous one.

Lemma 3.4. For every $\varepsilon>0$ and $\lambda>\lambda^{\times}$there exists a constant $b(\varepsilon)>0$, independent of $\lambda$, such that for every solution $(u, w) \in\left[Z_{\lambda}, Y\right]$ to $\left(\mathrm{Q}_{\lambda}\right)$ it holds that

$$
(u(x), w(x))>\min \left\{b(\varepsilon) \lambda^{1 / 2} \operatorname{dist}(x, \Gamma), \rho_{\delta / \gamma}^{+}-\varepsilon\right\}(1, \vartheta),
$$

with $\vartheta=1-\delta /(\gamma \beta)$. In particular there exists $b_{0}>0$ such that

$$
(u(x), w(x))>\min \left\{b_{0} \lambda^{1 / 2} \operatorname{dist}(x, \Gamma), \rho_{\sigma_{0}}^{+}\right\}(1, \vartheta) .
$$

Proof. Let $\lambda>\lambda^{\times}$be fixed and suppose $(u, w) \in\left[Z_{\lambda}, Y\right]$ is solution to $\left(\mathrm{Q}_{\lambda}\right)$. If $\rho_{\delta / \gamma}^{+}-\varepsilon \leq \tau$ then (13) holds with $b(\varepsilon)=b$ and $b$ as in the previous lemma.

Suppose $\rho_{\delta / \gamma}^{+}-\varepsilon>\tau$. Since $f(s)-(\delta / \gamma) s>0$ for all $s \in\left(\rho_{\delta / \gamma}^{-}, \rho_{\delta / \gamma}^{+}\right)$ there exists $\ell_{\varepsilon}>0$ such that

$$
f(s)-(\delta / \gamma) s>\ell_{\varepsilon}(s-\tau) \quad \text { for all } s \in\left[\tau, \rho_{\delta / \gamma}^{+}-\varepsilon\right] .
$$

From Lemma 3.3 it follows that $(u(x), w(x))>(\tau, \vartheta \tau)$ for all $x \in \Omega$ such that $\operatorname{dist}(x, \Gamma)>\lambda^{-1 / 2} \tau / b$. For subsolutions we need the function $e \geq 0$ satisfying

$$
\left\{\begin{aligned}
-\Delta e & =\mu e & & \text { in } B_{1} \\
e & =0 & & \text { on } \partial B_{1}
\end{aligned}\right.
$$

where $\mu$ is the principal eigenvalue and $B_{1}$ the unit ball in $\mathbb{R}^{N}$. We normalize $e$ such that $e(0)=1$. Let $\mu_{\varepsilon}=\mu / \ell_{\varepsilon}$ and

$$
\Omega^{\prime}:=\left\{y \in \Omega ; \operatorname{dist}(y, \Gamma)>\left(\sqrt{\mu_{\varepsilon}}+\tau / b\right) \lambda^{-1 / 2}\right\} .
$$

We fix $y \in \Omega^{\prime}$ and let $B:=B\left(y,\left(\mu_{\varepsilon} / \lambda\right)^{1 / 2}\right)$. For every $t \in[0,1]$ we define the functions $\left(U_{t}, W_{t}\right)$ on $\bar{B}$ by

$$
\begin{aligned}
& U_{t}(x):=\tau+t\left(\rho_{\delta / \gamma}^{+}-\varepsilon-\tau\right) e\left(\left(\lambda / \mu_{\varepsilon}\right)^{1 / 2}(y-x)\right), \\
& W_{t}(x):=\vartheta U_{t}(x) .
\end{aligned}
$$

Then $\mathcal{T}:=\left\{\left(U_{t}, W_{t}\right) ; t \in[0,1]\right\}$ is a family of subsolutions to the problem

$$
\left\{\begin{aligned}
-\Delta p & =\lambda(f(p)-\beta p+\beta q) & & \text { in } B, \\
-\Delta q & =\lambda(f(p)+M p-\alpha q) & & \text { in } B, \\
p & =u & & \text { on } \partial B \\
q & =w & & \text { on } \partial B .
\end{aligned}\right.
$$

Using the sweeping principle it follows that

$$
(u(y), w(y))>\left(U_{1}(y), W_{1}(y)\right)=\left(\rho_{\delta / \gamma}^{+}-\varepsilon, \vartheta\left(\rho_{\delta / \gamma}^{+}-\varepsilon\right)\right) .
$$


Since $y \in \Omega^{\prime}$ was arbitrary we have that

$$
(u(x), w(x))>\left(\rho_{\delta / \gamma}^{+}-\varepsilon, \vartheta\left(\rho_{\delta / \gamma}^{+}-\varepsilon\right)\right) \text { if } \operatorname{dist}(x, \Gamma)>\left(\mu_{\varepsilon}+\tau / b\right) \lambda^{-1 / 2} .
$$

Define $b(\varepsilon):=\tau\left(\tau / b+\sqrt{\mu_{\varepsilon}}\right)^{-1}$, and note that $\min \left\{b \lambda^{1 / 2} \operatorname{dist}(x, \Gamma), \tau\right\} \geq$ $b(\varepsilon) \lambda^{1 / 2} \operatorname{dist}(x, \Gamma)$ if $\operatorname{dist}(x, \Gamma) \leq\left(\sqrt{\mu_{\varepsilon}}+\tau / b\right) \lambda^{-1 / 2}$. Hence by Lemma 3.3

$$
(u(x), w(x))>b(\varepsilon) \lambda^{1 / 2} \operatorname{dist}(x, \Gamma)(1, \vartheta)
$$

for all $x$ with $\operatorname{dist}(x, \Gamma) \leq\left(\sqrt{\mu_{\varepsilon}}+\tau / b\right) \lambda^{-1 / 2}$. This proves (14) while (15) follows by choosing $b_{0}=b(\varepsilon)$ with $\varepsilon=\rho_{\delta / \gamma}^{+}-\rho_{\sigma_{0}}^{+}$.

The next lemma will be used in the proof of Theorem 2.3.

Lemma 3.5. Let $z_{0} \in C_{0}(\Omega)$ be nonnegative with $\max z_{0} \in\left(\rho_{\beta}^{-}, \rho_{\delta / \gamma}^{+}\right)$. There exists $\lambda_{z_{0}}>0$ such that if $(u, w)$ is a solution to $\left(\mathrm{Q}_{\lambda}\right)$ with $u \in$ $\left[z_{0}, \rho_{\delta / \gamma}^{+}\right]$and $\lambda>\lambda_{z_{0}}$ then $(u, w) \in\left[Z_{\lambda}, Y\right]$.

Proof. First note that if $u \in\left[z_{0}, \rho_{\delta / \gamma}^{+}\right]$then $(u, w) \in\left[\left(z_{0}, 0\right), Y\right]$. Let $x_{0} \in$ $\Omega$ be such that $z_{0}\left(x_{0}\right)=\max z_{0}$. Choose $\rho \in\left(\rho_{\beta}^{-}, \tau\right)$, where $\tau$ is as in Proposition 3.1, and $r_{0}>0$ such that $\rho<z_{0}(x) \leq u(x)$ for all $x \in B\left(x_{0}, r_{0}\right)$. Since $f(s)-\beta s>0$ for all $s \in\left(\rho_{\beta}^{-}, \rho_{\beta}^{+}\right)$there exists $\ell>0$ such that

$$
f(s)-\beta s>\ell(s-\rho) \quad \text { for all } s \in[\rho, \tau] .
$$

Let $e$ and $\mu$ be as (16) with $e(0)=1$. Suppose that

$$
\lambda>(\mu / \ell) r_{0}^{-2} \text {. }
$$

Then $r_{\lambda}:=r_{0}-\sqrt{\mu /(\lambda \ell)}>0$. Let $y \in B\left(x_{0}, r_{\lambda}\right)$ be fixed and define on $B=B(y, \sqrt{\mu /(\ell \lambda)}) \subset B\left(x_{0}, r_{0}\right)$,

$$
U_{t}(x)=\rho+t(\tau-\rho) e(\sqrt{\ell \lambda / \mu}(y-x)) .
$$

It holds that $\mathcal{T}:=\left\{\left(U_{t}, 0\right) ; t \in[0,1]\right\}$ is a family of subsolutions to (17) with $u_{\lambda}, w_{\lambda}$, instead of $u, w$. By a sweeping argument, starting with $\left(U_{0}, 0\right)$ one concludes that $(u(y), w(y))>\left(U_{1}(y), W_{1}(y)\right)=(\tau, 0)$. Since $y \in B\left(x_{0}, r_{\lambda}\right)$ was arbitrary we have that $(u, w) \geq(\tau, 0)$ on $B\left(x_{0}, r_{\lambda}\right)$.

Let $Z_{\lambda, i}^{x_{0}}, i=1,2$, denote the two components of $Z_{\lambda}^{x_{0}}$ defined in (12). The function $Z_{\lambda, 1}^{x_{0}}$ has support $\bar{B}\left(x_{0}, \sqrt{\lambda_{B} / \lambda}\right)$. Hence, if (19) is replaced by the stronger condition $\lambda>\left(\sqrt{\lambda_{B}}+\sqrt{\mu / \ell}\right)^{2} r_{0}^{-2}$, then $r_{\lambda}>\sqrt{\lambda_{B} / \lambda}$ and $(u, w)(x)>\left(Z_{\lambda, 1}^{x_{0}}, 0\right)$ for all $x \in \Omega$.

From this it follows that $(u, w) \in\left[Z_{\lambda}^{x_{0}}, Y\right]$. Indeed, using the fact that $Z_{\lambda}^{x_{0}}$ is a subsolution one has that $-\Delta\left(w-Z_{\lambda, 2}^{x_{0}}\right)+\alpha\left(w-Z_{\lambda, 2}^{x_{0}}\right) \geq 0$ in $\mathcal{D}^{\prime}(\Omega)$ sense.

As in the proof of Lemma 3.3 it now follows that $(u, w) \in\left[Z_{\lambda}, Y\right]$. 
3.2. The semilinear problem on the half space. In this section we consider the following problem

$$
\left\{\begin{aligned}
-\Delta U & =f(U)-\beta U+\beta W & & \text { in } \mathbb{R}_{+}^{N}, \\
-\Delta W & =f(U)+M U-\alpha W & & \text { in } \mathbb{R}_{+}^{N}, \\
U & =W=0 & & \text { on } \partial \mathbb{R}_{+}^{N} .
\end{aligned}\right.
$$

The main result which we prove is that there exists a positive solution $(U, W)$ to $(20)$ such that

$$
\lim _{x_{1} \rightarrow \infty}(U, W)\left(x_{1}, x^{\prime}\right)=\left(\rho_{\delta / \gamma}^{+}, \vartheta \rho_{\delta / \gamma}^{+}\right) \text {uniformly in } x^{\prime} \in \mathbb{R}^{N-1}
$$

with $\vartheta=1-\delta /(\gamma \beta)$. Moreover there exists only one such solution and $(U, W)\left(x_{1}, x^{\prime}\right)=(u, w)\left(x_{1}\right)$ where $(u, w)$ a solution to the problem

$$
\left\{\begin{array}{rlrl}
-u^{\prime \prime} & =f(u)-\beta u+\beta w & & \text { in } \mathbb{R}^{+}, \\
-w^{\prime \prime} & =f(u)+M u-\alpha w & & \text { in } \mathbb{R}^{+}, \\
u(0) & =0, \quad w(0)=0 & \\
u^{\prime}(0) & =\kappa, \quad w^{\prime}(0)=\nu, &
\end{array}\right.
$$

for some appropriate initial data $\kappa$ and $\nu$. It is standard that we have for every pair $(\kappa, \nu) \in \mathbb{R}^{2}$ at least locally a unique solution to (22) which can be continued to some maximum interval. We denote such a solution by $(u, w)_{\kappa, \nu}=\left(u_{\kappa, \nu}, w_{\kappa, \nu}\right)$. First we show that there exists a unique pair $(\kappa, \nu)$ such that the corresponding solution exists for all $r \in \mathbb{R}^{+}$, is positive and tends to $\left(\rho_{\delta / \gamma}^{+}, \vartheta \rho_{\delta / \gamma}^{+}\right)$at infinity. Some properties of this solution that are needed later, are also proven.

Proposition 3.6. Assume that $f$ satisfies Condition $\mathrm{A}^{*}$. Then there exists a unique pair $(\bar{\kappa}, \bar{\nu})$ such that the solution $(u, w)_{\bar{\kappa}, \bar{\nu}}$ to $(22)$ is positive and satisfies

$$
\lim _{r \rightarrow \infty}(u, w)_{\bar{\kappa}, \bar{\nu}}(r)=\left(\rho_{\delta / \gamma}^{+}, \vartheta \rho_{\delta / \gamma}^{+}\right)
$$

with $\vartheta=1-\delta /(\beta \gamma)$. Moreover $\bar{\kappa}>\bar{\nu}>0$ and $(u, w)_{\bar{\kappa}, \bar{\nu}}$ has the following properties:

(1) $0<(u, w)_{\bar{\kappa}, \bar{\nu}}(r)<\left(\rho_{\delta / \gamma}^{+}, \vartheta \rho_{\delta / \gamma}^{+}\right)$for all $r>0$;

(2) $u_{\bar{\kappa}, \bar{\nu}}(r)>w_{\bar{\kappa}, \bar{\nu}}(r)$ for all $r>0$;

(3) $\left(u^{\prime}, w^{\prime}\right)_{\bar{\kappa}, \bar{\nu}}(r)>(0,0)$ for all $r \in \mathbb{R}^{+}$and $\left(u^{\prime}, w^{\prime}\right)_{\bar{\kappa}, \bar{\nu}}(r) \rightarrow(0,0)$ as $r \rightarrow \infty$. 
The proof of this proposition consists of a number of lemmas. We also need to consider the following system

$$
\left\{\begin{array}{rlrl}
-u^{\prime \prime} & =f(u)-v & & \text { in } \mathbb{R}^{+}, \\
-v^{\prime \prime} & =\delta u-\gamma v & & \text { in } \mathbb{R}^{+}, \\
u(0) & =0, \quad v(0)=0, & & \\
u^{\prime}(0) & =\kappa, \quad v^{\prime}(0)=\eta . &
\end{array}\right.
$$

Again we denote solutions to $(24)$ by $(u, v)_{\kappa, \eta}$ with the understanding that the solutions are defined on a maximum interval. We point out the fact that for $\kappa, \nu \in \mathbb{R}$ it holds that the solution $(u, v)_{\kappa, \beta(\kappa-\nu)}$ to (24) is given by $\left(u_{\kappa, \nu}, \beta\left(u_{\kappa, \nu}-w_{\kappa, \nu}\right)\right)$ where $(u, w)_{\kappa, \nu}$ is the solution to $(22)$.

For a solution $(u, v)=(u, v)_{\kappa, \eta}$ to $(24)$ we have the following identity for all $r \geq 0$ :

$$
\left(\left(u^{\prime}\right)^{2}-\kappa^{2}\right)-\frac{1}{\delta}\left(\left(v^{\prime}\right)^{2}-\eta^{2}\right)=-2 \int_{0}^{u} f(s) d s+2 u v-\frac{\gamma}{\delta} v^{2} .
$$

Indeed, differentiating

$$
H(r):=u^{\prime}(r)^{2}-\frac{1}{\delta} v^{\prime}(r)^{2}+2 \int_{0}^{u(r)} f(s) d s-2 u(r) v(r)+\frac{\gamma}{\delta} v(r)^{2},
$$

(24) implies that $H^{\prime}(r)=0$ for all $r \geq 0$. Hence $H(r)=H(0)$ for all $r \geq 0$, which gives (25).

We shall often use the following one dimensional maximum principle, see e.g., [11, Theorem 2.9.2].

Lemma 3.7. If $g \in C^{2}[0,+\infty)$ is bounded, $g(0) \geq 0$ and $-g^{\prime \prime}+c g \supsetneqq 0$ with $c>0$, then $g(r)>0$ for all $r>0$. Moreover, if $g(0)=0$ then $g^{\prime}(0)>0$.

Our first lemma is on the derivatives of solutions to (22).

Lemma 3.8. Suppose that $(u, w)_{\kappa, \nu}$ is a solution to (22) with $\kappa, \nu>0$ and $(u, w)_{\kappa, \nu}(r)>(0,0)$ for all $r>0$. Then $\left(u^{\prime}, w^{\prime}\right)_{\kappa, \nu}(r)>(0,0)$ for all $r \geq 0$.

Proof. Since the system is quasimonotone this follows from a moving plane argument, similar to the method used by Gidas, Ni and Nirenberg [8]. See also [2] where a similar argument is used for a scalar equation.

Lemma 3.9. For a bounded solution $(u, w)_{\kappa, \nu}$ to $(22)$ with $u_{\kappa, \nu} \supsetneqq 0$ it holds that $0<\nu<\kappa$ and $0<w_{\kappa, \nu}(r)<u_{\kappa, \nu}(r)$ for all $r>0$.

Proof. Denote by $(u, w)$ the solution $(u, w)_{\kappa, \nu}$. Since $w$ is bounded and satisfies $-w^{\prime \prime}+\alpha w=f(u)+M u \supsetneqq 0$ with $w(0)=0$ we have by Lemma 3.7 that $\nu>0$ and $w(r)>0$ for all $r>0$. Let $\eta=\beta(\kappa-\nu)$. As observed earlier, the solution $(u, v)=(u, v)_{\kappa, \eta}$ to $(24)$ is given by $(u, \beta(u-w))$. Since $v$ is bounded with $v(0)=0$ and $-v^{\prime \prime}+\gamma v=\delta u \supsetneqq 0$ it holds again by Lemma 3.7 that $\eta>0$ and $v(r)>0$ for $r>0$. Hence $\kappa>\nu$ and $w(r)<u(r)$ for $r>0$. 
Lemma 3.10. If $(u, w)_{\kappa, \nu}$ is a positive solution to (22) such that (23) holds then

$$
\lim _{r \rightarrow \infty}\left(u^{\prime}, w^{\prime}\right)_{\kappa, \nu}(r)=0 .
$$

Proof. Define $u_{K}(r):=\rho_{\delta / \gamma}^{+}-u_{\kappa, \nu}(r+K)$ and $w_{K}(r):=\vartheta \rho_{\delta / \gamma}^{+}-w_{\kappa, \nu}(r+K)$. It holds that $u_{K}$ and $w_{K}$ converge uniformly to zero on $[0,1]$ as $K \rightarrow \infty$. From (22) we have that they remain bounded in $C^{2}[0,1]$. By interpolation $u_{K}, w_{K}$ converge to zero in $C^{1}[0,1]$. Therefore $\left(u^{\prime}, w^{\prime}\right)_{\kappa, \nu}(K)=$ $\left(u_{K}^{\prime}, w_{K}^{\prime}\right)(0) \rightarrow(0,0)$, as $K \rightarrow \infty$.

Let $(u, w)_{\kappa, \nu}$ be a solution to (22) for which (23) holds. Then $(u, v)_{\kappa, \eta}$ with $\eta=\beta(\kappa-\nu)$ is a solution to $(24)$ and $v_{\kappa, \eta}=\beta\left(u_{\kappa, \nu}-w_{\kappa, \nu}\right) \rightarrow(\delta / \gamma) \rho_{\delta / \gamma}^{+}$ as $r \rightarrow \infty$. Using Lemma 3.10 and letting $r \rightarrow \infty$ in (25) we obtain the following relationship between $\kappa$ and $\nu$ :

$$
\kappa^{2}-\frac{\beta^{2}}{\delta}(\kappa-\nu)^{2}=2 \int_{0}^{\rho_{\delta / \gamma}^{+}}\left(f(s)-\frac{\delta}{\gamma} s\right) d s .
$$

This will be used to prove the uniqueness of such solutions. Next we show that there exists initial data $(\bar{\kappa}, \bar{\nu})$ for which the corresponding solution to (22) is positive and satisfies (23).

Lemma 3.11. There exists $\bar{\kappa}, \bar{\nu} \in \mathbb{R}$ such that the solution $(u, w)_{\bar{\kappa}, \bar{\nu}}$ to $(22)$ satisfies (23). Moreover this solution is positive and $0<\bar{\nu}<\bar{\kappa}$.

Proof. We shall use super- and subsolutions and Lemma A.4 to find a positive solution to

$$
\left\{\begin{array}{rlrl}
-u^{\prime \prime} & =f(u)-\beta u+\beta w & & \text { in } \mathbb{R}^{+}, \\
-w^{\prime \prime} & =f(u)+M u-\alpha w & & \text { in } \mathbb{R}^{+}, \\
u(0) & =w(0)=0, &
\end{array}\right.
$$

satisfying (23). As a supersolution we take $\left(\rho_{\delta / \gamma}^{+}, \vartheta \rho_{\delta / \gamma}^{+}\right)$. We have to construct a nonzero subsolution. From a phaseplane analysis one sees that the initial value problem

$$
\left\{\begin{array}{l}
-u^{\prime \prime}=f(u)-\beta u \quad \text { in } \mathbb{R}^{+}, \\
u(0)=0, \\
u^{\prime}(0)=\left(2 J_{\beta}(0)\right)^{1 / 2},
\end{array}\right.
$$

with $J_{\beta}(0)>0$ defined in (2), has a solution $\tilde{u}$ with $\lim _{r \rightarrow \infty} \tilde{u}(r)=\rho_{\beta}^{+}$and $\tilde{u}^{\prime}(r)>0$ for all $r \geq 0$. Then $(\tilde{u}, 0)$ is a subsolution. By Lemma A.4 there exists a solution $(u, w)$ to $(27)$ such that $(0, \tilde{u})<(u, w)<\left(\rho_{\delta / \gamma}^{+}, \vartheta \rho_{\delta / \gamma}^{+}\right)$. At this stage we may choose either the maximal or minimal solution. In the next lemma we shall prove that they are equal. Let $(\bar{\kappa}, \bar{\nu}):=\left(u^{\prime}(0), w^{\prime}(0)\right)$. Then $(u, w)$ is the solution to $(22)$ with $(\kappa, \nu)=(\bar{\kappa}, \bar{\nu})$. 
It holds that $u(r), w(r)>0$ for all $r>0$. Indeed, $u(r) \geq \tilde{u}(r)>0$ and since $w$ is bounded with $-w^{\prime \prime}+\alpha w=f(u)+M u \supsetneqq 0$ and $w(0)=0$ we have by Lemma 3.7 that $w(r)>0$ for $r>0$ and that $w^{\prime}(0)=\nu>0$. By Lemma $3.9, \bar{\kappa}>\bar{\nu}>0$.

Lemma 3.8 shows that $u^{\prime}(r), w^{\prime}(r)>0$ for all $r>0$. In particular $\lim _{r \rightarrow \infty} u(r)=\rho$ and $\lim _{r \rightarrow \infty} w(r)=\tilde{\rho}$ exist. From the equations we find that

$$
-f(\rho)+\beta \rho-\beta \tilde{\rho}=-f(\rho)-M \rho+\alpha \tilde{\rho}=0 .
$$

From this one gets that $\tilde{\rho}=\vartheta \rho$ and that $f(\rho)=(\delta / \gamma) \rho$. Since $\rho>\rho_{\beta}^{+}$we have that $(\rho, \tilde{\rho})=\left(\rho_{\delta / \gamma}^{+}, \vartheta \rho_{\delta / \gamma}^{+}\right)$.

Our last lemma concerns the uniqueness part of Proposition 3.6.

Lemma 3.12. Let $(u, w)_{\kappa, \nu}$ be a positive solution to (22) such that (23) holds. Then $(\kappa, \nu)=(\bar{\kappa}, \bar{\nu})$ with $(\bar{\kappa}, \bar{\nu})$ as in Lemma 3.11 .

Proof. Let $(\tilde{u}, 0)$ be the subsolution of the previous lemma. First we show that the minimum and maximum solutions to (27) in the order interval

$$
\left[(\tilde{u}, 0),\left(\rho_{\delta / \gamma}^{+}, \vartheta \rho_{\delta / \gamma}^{+}\right)\right]
$$

are equal.

Let $(u, w)_{\kappa, \nu}$ be the minimal solution and $(u, w)_{\bar{\kappa}, \bar{\nu}}$ the maximal solution. It must hold that $\kappa \leq \bar{\kappa}$ and $\nu \leq \bar{\nu}$. If the solutions are not equal at least one of these inequalities must be strict. Suppose $\nu<\bar{\nu}$. By Lemma 3.9 we also have that $\kappa>\nu$ and $\bar{\kappa}>\bar{\nu}$ and by (26) that

$$
\frac{\delta-\beta^{2}}{\delta} \kappa^{2}+\frac{2 \beta^{2}}{\delta} \kappa \nu-\frac{\beta^{2}}{\delta} \nu^{2}=\frac{\delta-\beta^{2}}{\delta} \bar{\kappa}^{2}+\frac{2 \beta^{2}}{\delta} \bar{\kappa} \bar{\nu}-\frac{\beta^{2}}{\delta} \bar{\nu}^{2} .
$$

The function $x \mapsto\left(1-\left(\beta^{2} / \delta\right)\right) x^{2}+2\left(\beta^{2} / \delta\right) x \nu-\left(\beta^{2} / \delta\right) \nu^{2}$ is strictly increasing on $[\kappa, \bar{\kappa}]$ because it has derivative $2\left(\delta-\beta^{2}\right) x / \delta+2 \beta^{2} \nu / \delta$ which is strictly positive for $x \in[\kappa, \bar{\kappa}]$ since $\delta>\beta^{2}$. Hence

$$
\frac{\delta-\beta^{2}}{\delta} \kappa^{2}+\frac{2 \beta^{2}}{\delta} \kappa \nu-\frac{\beta^{2}}{\delta} \nu^{2} \leq \frac{\delta-\beta^{2}}{\delta} \bar{\kappa}^{2}+\frac{2 \beta^{2}}{\delta} \bar{\kappa} \nu-\frac{\beta^{2}}{\delta} \nu^{2} .
$$

The function $x \mapsto\left(1-\left(\beta^{2} / \delta\right)\right) \bar{\kappa}+2\left(\beta^{2} / \delta\right) \bar{\kappa} x-\left(\beta^{2} / \delta\right) x^{2}$ has derivative $2 \beta^{2} \bar{\kappa} \delta-2 \beta^{2} x / \delta$. Since the derivative is strictly positive on $[\nu, \bar{\nu}]$ it follows that

$$
\frac{\delta-\beta^{2}}{\delta} \kappa^{2}+\frac{2 \beta^{2}}{\delta} \kappa \nu-\frac{\beta^{2}}{\delta} \nu^{2}<\frac{\delta-\beta^{2}}{\delta} \bar{\kappa}^{2}+\frac{2 \beta^{2}}{\delta} \bar{\kappa} \bar{\nu}-\frac{\beta^{2}}{\delta} \bar{\nu}^{2},
$$

contradicting (28). If $\kappa<\bar{\kappa}$ we find a contradiction by the same argument. We conclude that $\kappa=\bar{\kappa}$ and $\nu=\bar{\nu}$ and that $(u, w)_{\kappa, \nu}=(u, w)_{\bar{\kappa}, \bar{\nu}}$.

It remains to show that any positive solution $(u, w)_{\kappa, \nu}$ for which (23) holds is in the order interval $\left[(\tilde{u}, 0),\left(\rho_{\delta / \gamma}^{+}, \vartheta \rho_{\delta / \gamma}^{+}\right)\right]$. First we show that $u_{\kappa, \nu}>\tilde{u}$. 
Suppose that $u_{\kappa, \nu}(r)>\rho_{\beta}^{+}$for all $r \geq R$. We define $u_{t}^{*}$ for $0 \leq t \leq R$ on $[0, R]$ by

$$
u_{t}^{*}(r):= \begin{cases}\tilde{u}(r-t) & \text { for } t \leq r \leq R \\ 0 & \text { for } 0 \leq r<t,\end{cases}
$$

with $\tilde{u}$ as in Lemma 3.11. Applying the sweeping principle with the subsolutions $\left\{\left(u_{t}^{*}, 0\right) ; 0 \leq t \leq R\right\}$ one finds that $(u, w)_{\kappa, \nu}(r)>(\tilde{u}(r), 0)=\left(u_{0}^{*}(r), 0\right)$ for $r \in(0, R)$. Hence $u_{\kappa, \nu}(r)>\tilde{u}(r)$ for $r>0$ and $(u, w)_{\kappa, \nu} \geq(\tilde{u}, 0)$. On the other hand, since $u_{\kappa, \nu}, w_{\kappa, \nu}$ are increasing by Lemma 3.8, it holds that $(u, w)_{\kappa, \nu}<\left(\rho_{\delta / \gamma}^{+}, \vartheta \rho_{\delta / \gamma}^{+}\right)$. Since there is only one solution in the order interval $\left[(\tilde{u}, 0),\left(\rho_{\delta / \gamma}^{+}, \vartheta \rho_{\delta / \gamma}^{+}\right)\right]$the uniqueness is proved.

Our main result concerning system (20) is the following.

Proposition 3.13. Assume that $f$ satisfies Condition $\mathrm{A}^{*}$. Then there exists a unique positive solution $(U, W)$ to (20) satisfying (21). This solution is given by

$$
(U, W)\left(x_{1}, x^{\prime}\right):=(u, w)_{\bar{\kappa}, \bar{\nu}}\left(x_{1}\right) \quad \text { for }\left(x_{1}, x^{\prime}\right) \in \mathbb{R}^{N-1},
$$

with $(u, w)_{\bar{\kappa}, \bar{\nu}}$ as in Lemma 3.11 .

Proof. Clearly (29) defines a positive solution to (20) satisfying (21). Suppose that $(U, W)$ is any positive solution satisfying (21). Define the functions

$$
\begin{aligned}
& (\underline{u}, \underline{w})\left(x_{1}\right):=\left(\sup _{x^{\prime} \in \mathbb{R}^{N-1}} U\left(x_{1}, x^{\prime}\right), \sup _{x^{\prime} \in \mathbb{R}^{N-1}} W\left(x_{1}, x^{\prime}\right)\right) ; \\
& \left.(\bar{u}, \bar{w})\left(x_{1}\right):=\inf _{x^{\prime} \in \mathbb{R}^{N-1}} U\left(x_{1}, x^{\prime}\right), \inf _{x^{\prime} \in \mathbb{R}^{N-1}} W\left(x_{1}, x^{\prime}\right)\right) .
\end{aligned}
$$

By Lemma A.5 $(\underline{u}, \underline{w})$ is a subsolution and $(\bar{u}, \bar{w})$ is a supersolution to (27). Moreover $(\underline{u}, \underline{w})<\left(\rho_{\delta / \gamma}^{+}, \vartheta \rho_{\delta / \gamma}^{+}\right)$. This follows by sweeping using the family of supersolutions $\left\{(t, \vartheta t): t \geq \rho_{\delta / \gamma}^{+}\right\}$.

Since $(\underline{u}, \underline{w})$ is a subsolution there exists a positive solution $(u, w)_{*}$ to (27) with $(\underline{u}, \underline{w}) \leq(u, w)_{*}<\left(\rho_{\delta / \gamma}^{+}, \vartheta \rho_{\delta / \gamma}^{+}\right)$. By Lemma 3.12 we have that $(u, w)_{*}=(u, w)_{\bar{\kappa}, \bar{\nu}}$.

Using a sweeping argument as in the proof of Lemma 3.12 it follows that $(\bar{u}, \bar{w})>(\tilde{u}, 0)$ with $\tilde{u}$ as in the proof of Lemma 3.11. Hence there exists a positive solution $(u, w)_{\circ}$ to $(27)$ with $(\tilde{u}, 0)<(u, w)_{\circ} \leq(\bar{u}, \bar{w})$ and by Lemma 3.12, $(u, w)_{\circ}=(u, w)_{\bar{\kappa}, \bar{\nu}}$. Hence $(\bar{u}, \bar{w})=(\underline{u}, \underline{w})$ which proves the uniqueness claim in the proposition.

3.3. The linearized problem on the halfspace. Let $(u, w)_{\bar{\kappa}, \bar{\nu}}$ be as in Proposition 3.6. In this paragraph we consider the following linear system:

$$
\left\{\begin{aligned}
-\bar{r} \Delta \Phi & =\left(f^{\prime}\left(u_{\bar{\kappa}, \bar{\nu}}\right)-\beta+\omega\right) \Phi+\beta \Psi-\bar{r} \omega \Phi & & \text { in } \mathbb{R}_{+}^{N}, \\
-\bar{r} \Delta \Psi & =\left(f^{\prime}\left(u_{\bar{\kappa}, \bar{\nu}}\right)+M\right) \Phi+(\omega-\alpha) \Psi-\bar{r} \omega \Psi & & \text { in } \mathbb{R}_{+}^{N}, \\
\Phi & =\Psi=0 & & \text { on } \partial \mathbb{R}_{+}^{N} .
\end{aligned}\right.
$$


Here $\alpha, \beta, M$ are as in (4), (3) and B1 respectively, $\omega>\max \{\alpha, M\}$ and $\bar{r} \in \mathbb{R}$. For this problem we have the following result of Liouville type.

Proposition 3.14. Suppose that $\bar{r} \geq 1$ and that $(\Phi, \Psi)$ is a bounded positive solution to (30). Then $(\Phi, \Psi) \equiv(0,0)$.

This proposition will be a consequence of the following lemma.

Lemma 3.15. Suppose $\varphi, \psi \in C[0,+\infty)$ are bounded with $\varphi, \psi \geq 0, \varphi(0)=$ $\psi(0)=0$ and it holds that

$$
\begin{aligned}
-\varphi^{\prime \prime} & \leq f^{\prime}\left(u_{\bar{\kappa}, \bar{\nu}}\right) \varphi-\beta \varphi+\beta \psi, \\
-\psi^{\prime \prime} & \leq f^{\prime}\left(u_{\bar{\kappa}, \bar{\nu}}\right) \varphi+M \varphi-\alpha \psi,
\end{aligned}
$$

in $\mathcal{D}^{\prime}(0, \infty)$-sense. Then $\varphi\left(x_{1}\right)=\psi\left(x_{1}\right)=0$ for all $x_{1} \geq 0$.

Proof. We set $(p, q):=\left(u^{\prime}, w^{\prime}\right)_{\bar{\kappa}, \bar{\nu}}$ and recall that $p, q>0$ on $[0, \infty)$. Without loss of generality we assume that $\varphi, \psi \leq 1$. We argue by contradiction and suppose that $(\varphi, \psi) \neq(0,0)$. First we observe that if there exists $K>0$ such that $\varphi\left(x_{1}\right)=\psi\left(x_{1}\right)=0$ for all $x_{1} \geq K$ then by a sweeping argument on $[0, K]$ with the family $\{(t p, t q) ; t \geq 0\}$ of supersolutions it follows that $\varphi\left(x_{1}\right)=\psi\left(x_{1}\right)=0$ for all $x_{1} \in[0, K]$. This is in contradiction with our assumption.

Now let $K>0$ and $\varepsilon>0$ be such that that

$$
f^{\prime}\left(u_{\bar{\kappa}, \bar{\nu}}\left(x_{1}\right)\right)<-\varepsilon \text { for all } x_{1} \geq K,
$$

and note that also

$$
f^{\prime}\left(u_{\bar{\kappa}, \bar{\nu}}\left(x_{1}\right)\right)+M-\alpha<-\varepsilon \text { for all } x_{1} \geq K .
$$

By our first observation we may assume that

$$
R(K):=\max \{\varphi(K) / p(K), \psi(K) / q(K)\}>0 .
$$

We define the following functions on $[K, \infty)$ :

$$
\begin{aligned}
S_{t}\left(x_{1}\right) & =\varphi\left(x_{1}\right)-e^{\sqrt{\varepsilon}\left(x_{1}-t\right)}, \\
T_{t}\left(x_{1}\right) & =\psi\left(x_{1}\right)-e^{\sqrt{\varepsilon}\left(x_{1}-t\right)}, \\
R_{t}\left(x_{1}\right) & =\max \left\{S_{t}\left(x_{1}\right) / p\left(x_{1}\right), T_{t}\left(x_{1}\right) / q\left(x_{1}\right)\right\}
\end{aligned}
$$

for $t \geq K$. It holds that

$$
-S_{t}^{\prime \prime} \leq\left(f^{\prime}\left(u_{\bar{\kappa}, \bar{\nu}}\right)-\beta\right) S_{t}+\beta T_{t},
$$

and

$$
-T_{t}^{\prime \prime} \leq\left(f^{\prime}\left(u_{\bar{\kappa}, \bar{\nu}}\right)+M\right) S_{t}-\alpha T_{t}
$$

in $\mathcal{D}^{\prime}(K, \infty)$-sense. For $t>K$ let $m_{t}=\sup _{x_{1} \in[K, t]} R_{t}\left(x_{1}\right)$. By the maximum principle one has that $m_{t}=R_{t}(K)$ for $t$ large enough. Indeed, since for $\omega$ large enough, it holds in $\mathcal{D}^{\prime}(K, t)$-sense that

$$
-\left(S_{t}-m_{t} p\right)^{\prime \prime}+\omega\left(S_{t}-m_{t} p\right) \leq 0
$$


and

$$
-\left(T_{t}-m_{t} q\right)^{\prime \prime}+\alpha\left(T_{t}-m_{t} q\right) \leq 0,
$$

we see that $m_{t}$ must be attained in $K$ or in $t$. Since $R_{t}(t) \leq 0$ and $R_{t}(K)>0$, if $t$ is large enough, we conclude that $m_{t}=R_{t}(K)$. Now let $x_{1} \in[K, \infty)$ be fixed. Then for all $t>x_{1}$ large we have

$$
\begin{aligned}
R\left(x_{1}\right) & =\max \left\{\varphi\left(x_{1}\right) / p\left(x_{1}\right), \psi\left(x_{1}\right) / q\left(x_{1}\right)\right\} \\
& \leq R_{t}(K)+\max \left\{e^{\sqrt{\varepsilon}\left(x_{1}-t\right)} / p\left(x_{1}\right), e^{\sqrt{\varepsilon}\left(x_{1}-t\right)} / q\left(x_{1}\right)\right\} .
\end{aligned}
$$

Letting $t \rightarrow \infty$ we deduce that $R\left(x_{1}\right) \leq R(K)$ and hence $R$ attains its maximum on $[K, \infty)$ in $x_{1}=K$. Consequently $\sup _{x_{1} \in[0, \infty)} R\left(x_{1}\right)$ is attained in some point $r_{0} \in(0, K]$. But this is in contradiction to the maximum principle. Indeed, in a similar way as above, one sees that $R\left(x_{1}\right)$ must attain its maximum on [0,K+1] either in 0 or in $K+1$ and not in $K$.

To see how Proposition 3.14 follows from this lemma, we define

$$
\begin{aligned}
& \varphi\left(x_{1}\right):=\sup \left\{\Phi\left(x_{1}, x^{\prime}\right) ; x^{\prime} \in \mathbb{R}^{N-1}\right\}, \\
& \psi\left(x_{1}\right):=\sup \left\{\Psi\left(x_{1}, x^{\prime}\right) ; x^{\prime} \in \mathbb{R}^{N-1}\right\} .
\end{aligned}
$$

Then by Lemma A.5, $\varphi, \psi \in C[0,+\infty)$ with $\varphi(0)=\psi(0)=0$ and in $\mathcal{D}^{\prime}\left(\mathbb{R}_{+}^{N}\right)$-sense

$$
\begin{aligned}
-\varphi^{\prime \prime} & \leq \frac{1}{\bar{r}}\left(f^{\prime}\left(u_{\bar{\kappa}, \bar{\nu}}\right)-\beta+\omega\right) \varphi+\frac{1}{\bar{r}} \beta \psi-\omega \varphi \\
-\psi^{\prime \prime} & \leq \frac{1}{\bar{r}}\left(f^{\prime}\left(u_{\bar{\kappa}, \bar{\nu}}\right)+M\right) \varphi+\frac{1}{\bar{r}}(\omega-\alpha) \psi-\omega \psi .
\end{aligned}
$$

Since $\bar{r} \geq 1$ we deduce that

$$
\begin{aligned}
-\varphi^{\prime \prime} & \leq\left(f^{\prime}\left(u_{\bar{\kappa}, \bar{\nu}}\right)-\beta+\omega\right) \varphi+\beta \psi-\omega \varphi \\
& =\left(f^{\prime}\left(u_{\bar{\kappa}, \bar{\nu}}\right)-\beta+\omega\right) \varphi+\beta \psi
\end{aligned}
$$

and

$$
\begin{aligned}
-\psi^{\prime \prime} & \leq\left(f^{\prime}\left(u_{\bar{\kappa}, \bar{\nu}}\right)+M\right) \varphi+(\omega-\alpha) \psi-\omega \psi \\
& =\left(f^{\prime}\left(u_{\bar{\kappa}, \bar{\nu}}\right)+M\right) \varphi-\alpha \psi .
\end{aligned}
$$

By the lemma $(\varphi, \psi)\left(x_{1}\right)=0$ for $x_{1} \geq 0$ and hence also $(\Phi, \Psi)(x)=(0,0)$ on $\mathbb{R}_{+}^{N}$.

\section{Proofs of the main results.}

4.1. Proof of Theorem 2.1. From now on we assume that $\Gamma$ is $C^{3}$. We begin by defining some operators. Recall that $X$ denotes the space $C(\bar{\Omega}) \times$ $C(\bar{\Omega})$ and let $C_{0}^{1}(\bar{\Omega})=\left\{u \in C^{1}(\bar{\Omega}) ; u(x)=0\right.$ for $\left.x \in \Gamma\right\}$. For $k, \lambda>0$ 
define $\left(-\lambda^{-1} \Delta+k\right)_{0}^{-1}: C(\bar{\Omega}) \rightarrow C_{0}^{1}(\bar{\Omega})$ by $u=\left(-\lambda^{-1} \Delta+k\right)_{0}^{-1} g$ with $u \in C_{0}^{1}(\bar{\Omega})$ the unique function satisfying

$$
\left\{\begin{aligned}
-\lambda^{-1} \Delta u+k u & =g & & \text { in } \mathcal{D}^{\prime}(\Omega) \text {-sense } \\
u & =0 & & \text { on } \Gamma .
\end{aligned}\right.
$$

Let $j$ be the embedding of $C_{0}^{1}(\bar{\Omega}) \times C_{0}^{1}(\bar{\Omega})$ in $X$ and define the operator $K_{k, \lambda}: X \rightarrow X$ by

$$
K_{k, \lambda}\left(\begin{array}{l}
g \\
h
\end{array}\right)=j \circ\left(\begin{array}{cc}
\left(-\lambda^{-1} \Delta+k\right)_{0}^{-1} & 0 \\
0 & \left(-\lambda^{-1} \Delta+k\right)_{0}^{-1}
\end{array}\right)\left(\begin{array}{l}
g \\
h
\end{array}\right) .
$$

Since $j$ is compact and $\left(-\lambda^{-1} \Delta+k\right)_{0}^{-1}$ is continuous, $K_{k, \lambda}$ is a compact linear map on $X$. We shall also use the fact that $\left\|K_{k, \lambda}\right\|_{\mathcal{L}(X)}$ is uniformly bounded in $\lambda$. This follows from the fact that

$$
\left\|\left(-\lambda^{-1} \Delta+k\right)_{0}^{-1} g\right\|_{\infty} \leq \frac{1}{k}\|g\|_{\infty}
$$

for every $g \in C(\bar{\Omega})$. We fix $\omega>\max \{\alpha, M\}$. For a function $u \in C(\bar{\Omega})$ and $\lambda>0$ the we define the operators $M_{u}, T_{u, \lambda} \in \mathcal{L}(X)$ by

$$
M_{u}\left(\begin{array}{c}
h \\
g
\end{array}\right)=\left(\begin{array}{cc}
f^{\prime}(u)-\beta & \beta \\
f^{\prime}(u)+M & -\alpha
\end{array}\right)\left(\begin{array}{c}
h \\
g
\end{array}\right),
$$

and

$$
T_{u, \lambda}:=K_{\omega, \lambda}\left(M_{u}+\omega I\right) .
$$

Operators of this kind were studied extensively in [23]. If $u \in\left[0, \rho_{\delta / \gamma}^{+}\right]$then $T_{u, \lambda}$ is a positive irreducible compact operator on $X$, see [23, Lemma 1.3]. Moreover, $T_{u, \lambda}$ has a positive spectral radius (see e.g., [18]) which we denote by $r\left(T_{u, \lambda}\right)$. By the Krein-Rutman Theorem (see e.g., [1, Theorem 3.1]), $r\left(T_{u, \lambda}\right)$ is an eigenvalue of $T_{u, \lambda}$ to which a positive eigenfunction pertains. In the next lemma we prove that for $\lambda$ large enough it holds for every solution $(u, w) \in\left[Z_{\lambda}, Y\right]$ that $r\left(T_{u, \lambda}\right)<1$.

Lemma 4.1. There exists $\lambda^{\star}>\lambda^{\star}$ such that for all $\lambda>\lambda^{\star}$ and every solution $(u, w) \in\left[Z_{\lambda}, Y\right]$ to $\left(\mathrm{Q}_{\lambda}\right)$ the corresponding operator $T_{u, \lambda}$ has spectral radius $r\left(T_{u, \lambda}\right)<1$.

Proof. We prove the lemma by a contradiction argument. Assume that it does not hold. Then there exist a sequence $\left\{\lambda_{n}\right\}_{n=1}^{\infty}$ with $\lambda^{\times}<\lambda_{n} \rightarrow \infty$ and solutions $\left(u_{n}, w_{n}\right):=\left(u_{\lambda_{n}}, w_{\lambda_{n}}\right) \in\left[Z_{\lambda_{n}}, Y\right]$ to $\left(\mathrm{Q}_{\lambda}\right)$ with $\lambda=\lambda_{n}$ such that $r_{n} \geq 1$, with $r_{n}$ denoting the spectral radius of $T_{n}:=T_{u_{n}, \lambda_{n}}$. Let $\left(\varphi_{n}, \psi_{n}\right) \in X$ be the positive eigenfunction pertaining to $r_{n}$. We normalize the eigenfunction such that $\max \varphi_{n}=1$. This can be done since $\varphi_{n}=0$ 
implies that $\psi_{n}=0$, a contradiction because $\left(\varphi_{n}, \psi_{n}\right)$ is an eigenfunction. It holds that

$$
\left\{\begin{aligned}
-r_{n} \lambda_{n}^{-1} \Delta \varphi_{n} & =\left(f^{\prime}\left(u_{n}\right)+\omega-\beta\right) \varphi_{n}+\beta \psi_{n}-r_{n} \omega \varphi_{n} & & \text { in } \Omega \\
-r_{n} \lambda_{n}^{-1} \Delta \psi_{n} & =\left(f^{\prime}\left(u_{n}\right)+M\right) \varphi_{n}+(\omega-\alpha) \psi_{n}-r_{n} \omega \psi_{n} & & \text { in } \Omega \\
\varphi_{n} & =\psi_{n}=0 & & \text { on } \partial \Omega .
\end{aligned}\right.
$$

Because operator norms $\left\|T_{n}\right\|_{\mathcal{L}(X)}$ are uniformly bounded it follows from

$$
r_{n}=\varlimsup_{k \rightarrow \infty}\left(\left\|T_{n}^{k}\right\|_{\mathcal{L}(X)}\right)^{1 / k} \leq\left\|T_{n}\right\|_{\mathcal{L}(X)}
$$

that the sequence $\left\{r_{n}\right\}_{n=1}^{\infty}$ is bounded. By going over to a subsequence, still denoted by $\left\{r_{n}\right\}_{n=1}^{\infty}$, we can assume that $r_{n} \rightarrow \bar{r} \geq 1$. With $\theta_{n}:=\beta\left(\varphi_{n}-\psi_{n}\right)$ one has that

$$
\left\{\begin{aligned}
-r_{n} \lambda_{n}^{-1} \Delta \theta_{n} & =\delta \varphi_{n}-\gamma \theta_{n}+\left(1-r_{n}\right) \omega \theta_{n} & & \text { in } \Omega, \\
\theta_{n} & =0 & & \text { on } \partial \Omega .
\end{aligned}\right.
$$

This shows that $\varphi_{n} \geq \psi_{n}$ and hence $-r_{n} \lambda_{n}^{-1} \Delta \varphi_{n} \leq f^{\prime}\left(u_{n}\right) \varphi_{n}$. Using estimate (15) in Lemma 3.4 we have for all $x \in \Omega$ with $\operatorname{dist}(x, \Gamma)>b_{0}^{-1} \lambda_{n}^{-1 / 2} \rho_{\sigma_{0}}^{+}$ that $f^{\prime}\left(u_{n}(x)\right) \leq 0$ and consequently

$$
-\Delta \varphi_{n} \leq 0 \quad \text { in } \quad\left\{x \in \Omega ; \operatorname{dist}(x, \Gamma)>b_{0}^{-1} \lambda_{n}^{-1 / 2} \rho_{\sigma_{0}}^{+}\right\} .
$$

Hence $\varphi_{n}$ attains its maximum in a point $\bar{x}_{n}$ with $\operatorname{dist}\left(\bar{x}_{n}, \Gamma\right) \leq b_{0}^{-1} \lambda_{n}^{-1 / 2} \rho_{\sigma_{0}}^{+}$. Let $\bar{x}_{\Gamma, n} \in \Gamma$ be such that $\left|\bar{x}_{n}-\bar{x}_{\Gamma, n}\right|=\operatorname{dist}\left(\bar{x}_{\Gamma, n}, \Gamma\right)$. By going over to a subsequence we can assume that $\bar{x}_{\Gamma, n} \rightarrow \bar{x} \in \Gamma$.

By a blow-up argument around $\bar{x}$, similar to the argument in [4], one constructs $U, W, \Phi, \Psi \in C^{2}\left(\mathbb{R}_{+}^{N}\right) \cap C\left(\overline{\mathbb{R}_{+}^{N}}\right)$ such that $(U, W)$ satisfies

$$
\left\{\begin{aligned}
-\Delta U & =f(U)-\beta U+\beta W & & \text { in } \mathbb{R}_{+}^{N}, \\
-\Delta W & =f(U)+M U-\alpha W & & \text { in } \mathbb{R}_{+}^{N}, \\
U & =W=0 & & \text { on } \partial \mathbb{R}_{+}^{N},
\end{aligned}\right.
$$

and $(\Phi, \Psi)$ satisfies

$$
\left\{\begin{aligned}
-\bar{r} \Delta \Phi & =\left(f^{\prime}(U)+\omega-\beta\right) \Phi+\beta \Psi-\bar{r} \omega \Phi & & \text { in } \mathbb{R}_{+}^{N}, \\
-\bar{r} \Delta \Psi & =\left(f^{\prime}(U)+M\right) \Phi+(\omega-\alpha) \Psi-\bar{r} \omega \Psi & & \text { in } \mathbb{R}_{+}^{N}, \\
\Phi & =\Psi=0 & & \text { on } \partial \mathbb{R}_{+}^{N} .
\end{aligned}\right.
$$

The normalization $\max \phi_{n}=1$ leads to $\sup \Phi=1$. Furthermore, using the uniform estimate (14) it follows that

$$
\lim _{x_{1} \rightarrow \infty}(U, W)\left(x_{1}, x^{\prime}\right)=\left(\rho_{\delta / \gamma}^{+}, \vartheta \rho_{\delta / \gamma}^{+}\right) \quad \text { uniformly in } x^{\prime} \in \mathbb{R}^{N-1} .
$$


Hence, by Proposition 3.13, $(U, W)\left(x_{1}, x^{\prime}\right)=(u, w)_{\bar{\kappa}, \bar{\nu}}\left(x_{1}\right)$. Then $(\Phi, \Psi)$ is a bounded positive solution to (30) with $\bar{r} \geq 1$. By Proposition 3.14, $(\Phi, \Psi) \equiv(0,0)$, in contradiction with $\sup \Phi=1$.

We shall use this lemma to prove that there can be at most one solution to $\left(\mathrm{Q}_{\lambda}\right)$ in the order interval $\left[Z_{\lambda}, Y\right]$. First we define the operator $H_{\lambda}: X \rightarrow X$

$$
H_{\lambda}:=K_{\omega, \lambda}(F+\omega I),
$$

where $F: X \rightarrow X$ is defined by

$$
F\left(\begin{array}{c}
u \\
w
\end{array}\right)=\left(\begin{array}{c}
f(u)-\beta u+\beta w \\
f(u)+M u-\alpha w
\end{array}\right) .
$$

We shall show that $H_{\lambda}$ has at most one fixed point in $\left[Z_{\lambda}, Y\right]$. In order to use the Leray-Schauder degree we have to consider the fixed point problem

$$
H_{\lambda}(u, w)=(u, w)
$$

in an appropriate space.

Let $\mu$ be the principal eigenvalue and $e \in C^{1}(\bar{\Omega}) \cap C^{2}(\Omega)$ the corresponding eigenfunction to the problem

$$
\left\{\begin{aligned}
-\Delta e & =\mu e & & \text { in } \Omega, \\
e & =0 & & \text { on } \Gamma .
\end{aligned}\right.
$$

We normalize $e$ such that $\max e=1$. Following Amann [1] we define

$$
C_{e}(\bar{\Omega}):=\{u \in C(\bar{\Omega}) ; \exists t>0 \text { such that }|u| \leq t e\},
$$

equipped with the norm $\|u\|_{e}=\inf \{t>0 ;-t e \leq u \leq t e\}$. It holds that $C_{e}(\bar{\Omega})$ is a Banach space, in fact a Banach lattice, with closed unit ball $\{u \in C(\bar{\Omega}) ;-e \leq u \leq e\}$. Let $X_{e}=C_{e}(\bar{\Omega}) \times C_{e}(\bar{\Omega})$. Order intervals in $X_{e}$ will be denoted by $[\cdot, \cdot]_{e}$. Let $j_{1}, j_{2}$ be the embeddings of $X_{e}$ in $X$ and $C_{0}^{1}(\bar{\Omega}) \times C_{0}^{1}(\bar{\Omega})$ in $X_{e}$ respectively and define $H_{\lambda}^{e}: X_{e} \rightarrow X_{e}$ by

$$
H_{\lambda}^{e}:=j_{2} \circ\left(\begin{array}{cc}
\left(-\lambda^{-1} \Delta+\omega\right)_{0}^{-1} & 0 \\
0 & \left(-\lambda^{-1} \Delta+\omega\right)_{0}^{-1}
\end{array}\right) \circ(F+\omega I) \circ j_{1} .
$$

We recall that $\left(-\lambda^{-1} \Delta+\omega\right)_{0}^{-1}$ was defined as an operator from $C(\bar{\Omega})$ into $C_{0}^{1}(\bar{\Omega})$. We note that $(u, w)$ is a fixed point of $H_{\lambda}^{e}$ if and only if $j_{1}(u, w)$ is a fixed point of $H_{\lambda}$. Hence it suffices to show that $H_{\lambda}^{e}$ has a unique fixed point in $\left[Z_{\lambda}, Y\right] \cap X_{e}$.

It also holds for $\left(u_{i}, w_{i}\right) \in X$ with $\left(u_{1}, w_{1}\right)<\left(u_{2}, w_{2}\right)$ that

$$
H_{\lambda}\left(u_{1}, w_{1}\right)<H_{\lambda}\left(u_{2}, w_{2}\right) \text {. }
$$

In fact $H_{\lambda}\left(u_{2}, w_{2}\right)-H_{\lambda}\left(u_{1}, w_{1}\right)$ is an element of the interior of the positive cone of $X_{e}$, or equivalently, there exists $t>0$ such that

$$
H_{\lambda}\left(u_{2}, w_{2}\right)-H_{\lambda}\left(u_{1}, w_{1}\right) \geq(t e, t e) .
$$


Since neither $Z_{\lambda}$ nor $Y$ are fixed points of $H_{\lambda}$ we find, using (39), that any fixed point $(u, w) \in\left[Z_{\lambda}, Y\right]$ satisfies

$$
Z_{\lambda}^{*}:=H_{\lambda} Z_{\lambda}<(u, w)<H_{\lambda} Y=: Y^{*} .
$$

From (40) we even have the stronger result that $(u, w) \in \operatorname{int}\left[Z_{\lambda}^{*}, Y^{*}\right]_{e}$, the

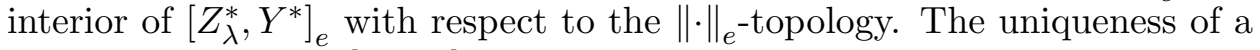
fixed point of $H_{\lambda}$ in $\left[Z_{\lambda}, Y\right]$ for $\lambda \geq \lambda^{\star}$ then follows from the next lemma.

Lemma 4.2. For every $\lambda>\lambda^{\star}$ there exists a unique fixed point of $H_{\lambda}^{e}$ in int $\left[Z_{\lambda}^{*}, Y^{*}\right]_{e}$.

Proof. We have for every $\lambda>\lambda^{\star}$ that there exists at least one solution to $\left(\mathrm{Q}_{\lambda}\right)$ in the order interval $\left[Z_{\lambda}, Y\right]$ which, as we observed, is a fixed point of $H_{\lambda}^{e}$ and $(u, w) \in \operatorname{int}\left[Z_{\lambda}^{*}, Y^{*}\right]_{e}$. To show that this is the only solution, we shall use a degree argument.

Suppose $(u, w) \in \operatorname{int}\left[Z_{\lambda}^{*}, Y^{*}\right]_{e}$, with $\lambda>\lambda^{\star}$, is a fixed point of $H_{\lambda}^{e}$. The operator $H_{\lambda}^{e}$ is differentiable and $T_{u, \lambda}^{e}:=d H_{\lambda}^{e}(u, w) \in \mathcal{L}\left(X_{e}\right)$ given by

$$
T_{u, \lambda}^{e}=j_{2} \circ\left(\begin{array}{cc}
\left(-\lambda^{-1} \Delta+\omega\right)_{0}^{-1} & 0 \\
0 & \left(-\lambda^{-1} \Delta+\omega\right)_{0}^{-1}
\end{array}\right) \circ\left(M_{u}+\omega I\right) \circ j_{1},
$$

with $M_{u}$ as defined in (34). From Lemma 4.1 we have that the spectral radius $r\left(T_{u, \lambda}^{e}\right)<1$. Indeed $\mu$ is an eigenvalue of $T_{u, \lambda}^{e}$ if and only if $\mu$ is an eigenvalue of $T_{u, \lambda}$. Since $r\left(T_{u, \lambda}\right)>0$ it holds that $r\left(T_{u, \lambda}^{e}\right)>0$. But $T_{u, \lambda}^{e}$ is a positive compact operator and hence $r\left(T_{u, \lambda}^{e}\right)$ is an eigenvalue of $T_{u, \lambda}^{e}$ to which a positive eigenfunction pertains. This implies that $r\left(T_{u, \lambda}^{e}\right)=r\left(T_{u, \lambda}\right)<1$.

In particular 1 is not an eigenvalue of $T_{u, \lambda}^{e}$ and consequently the index of the fixed point $(u, w)$ is well defined with

$$
\operatorname{index}(u, w)=1,
$$

see $[\mathbf{1 7}, \mathrm{p} .66]$. Using the homotopy invariance of the degree and the fact that int $\left[Z_{\lambda}^{*}, Y^{*}\right]_{e}$ is convex, it follows that

$$
\text { degree }\left(I-H_{u, \lambda}^{e}, \operatorname{int}\left[Z_{\lambda}^{*}, Y^{*}\right]_{e}, 0\right)=1 .
$$

Indeed, let $\bar{z} \in \operatorname{int}\left[Z_{\lambda}^{*}, Y^{*}\right]_{e}$ be arbitrary and define the homotopy

$$
G_{t}=(1-t)(I-\bar{z})+t\left(I-H_{u, \lambda}^{e}\right) \text {. }
$$

It holds that $G_{t} z=0$ if and only if $z=(1-t) \bar{z}+t H_{u, \lambda}^{e} z$ and hence $z \in$ $\operatorname{int}\left[Z_{\lambda}^{*}, Y^{*}\right]_{e}$. Since $G_{t}$ has no zeros on the boundary int $\left[Z_{\lambda}^{*}, Y^{*}\right]_{e}$ we have that

$$
\text { degree }\left(G_{1}, \operatorname{int}\left[Z_{\lambda}^{*}, Y^{*}\right]_{e}, 0\right)=\operatorname{degree}\left(G_{0}, \operatorname{int}\left[Z_{\lambda}^{*}, Y^{*}\right]_{e}, 0\right)=1 .
$$

By the additivity property of the degree we see that $H_{\lambda}^{e}$ can have at most one fixed point in int $\left[Z_{\lambda}^{*}, Y^{*}\right]_{e}$. 
The proof of Theorem 2.1 can now be completed. For all $\lambda>\lambda^{\star}$ we have a unique solution $\tilde{\Lambda}(\lambda)=\left(u_{\lambda}, w_{\lambda}\right) \in \operatorname{int}\left[Z_{\lambda}^{*}, Y^{*}\right]_{e} \subset\left[Z_{\lambda}, Y\right]$ to $\left(\mathrm{Q}_{\lambda}\right)$.

Using the Implicit Function Theorem we have that $\tilde{\Lambda} \in C^{1}\left(\left(\lambda^{\star},+\infty\right), X_{e}\right)$. Indeed the operator $(\lambda,(u, w)) \longmapsto(u, w)-H^{e}(\lambda,(u, w))$ is $C^{1}$ and the derivative with respect to $(u, w)$ is given by $I-T_{u, \lambda}^{e}$. For fixed $\lambda_{0}>\lambda^{\star}$ it holds that $I-T_{u, \lambda_{0}}^{e} \in \operatorname{Isom}\left(X_{e}\right)$. By the Implicit Function Theorem the solution set of $H^{e}(\lambda,(u, w))=0$ consists in a neighbourhood of $\lambda_{0}$ of a $C^{1}$-curve, parameterized by $\lambda$. By uniqueness of solutions in int $\left[Z_{\lambda}^{*}, Y^{*}\right]_{e}$ we have that this curve is in a neighbourhood of $\left(\lambda_{0}, u_{\lambda_{0}}, w_{\lambda_{0}}\right)$ given by $\tilde{\Lambda}$. Since this can be done for every $\lambda>\lambda^{\star}$ we have that $\tilde{\Lambda}$ in $C^{1}\left(\left(\lambda^{\star},+\infty\right), X_{e}\right)$. Using a bootstrap argument one proves that $\tilde{\Lambda} \in C^{1}\left(\left(\lambda^{\star},+\infty\right), C^{2}(\bar{\Omega}) \times C^{2}(\bar{\Omega})\right)$.

Finally we define $\Lambda(\lambda):=\left(u_{\lambda}, \beta\left(u_{\lambda}-w_{\lambda}\right)\right)$. Then $\Lambda(\lambda)$ is a solution to $\left(\mathrm{P}_{\lambda}\right)$ for all $\lambda>\lambda^{\star}$ and $\Lambda \in C^{1}\left(\left(\lambda^{\star}, \infty\right), C^{2}(\bar{\Omega}) \times C^{2}(\bar{\Omega})\right)$.

4.2. Proof of Theorems 2.2 and 2.3. In this section we assume that the conditions of Theorem 2.1 hold. We define the operator $B_{\lambda}: D\left(B_{\lambda}\right) \rightarrow X$ by

$$
D\left(B_{\lambda}\right):=\{(u, w) \in X ;(\Delta u, \Delta w) \in X\},
$$

with $\Delta u$ and $\Delta w$ in distributional sense,

$$
B_{\lambda}:=L_{\lambda}-M_{u_{\lambda}}
$$

with

$$
L_{\lambda}:=\left(\begin{array}{cc}
-\lambda^{-1} \Delta & 0 \\
0 & -\lambda^{-1} \Delta
\end{array}\right)
$$

and $M_{u_{\lambda}}$ as defined in (34).

Lemma 4.3. For all $\lambda>\lambda^{\star}$, with $\lambda^{\star}$ as in Lemma 4.1, the operator $B_{\lambda}$ is invertible and $B_{\lambda}^{-1} \in \mathcal{L}(X)$ is a positive compact operator with a positive spectral radius $r_{\lambda}=r\left(B_{\lambda}^{-1}\right)$. Moreover $r_{\lambda}$ is an eigenvalue of $B_{\lambda}^{-1}$ with a corresponding positive eigenfunction.

Proof. Denote by $T_{\lambda}$ and $M_{\lambda}$ the operators $T_{u_{\lambda}}$ and $M_{u_{\lambda}}$ respectively. Since $\lambda>\lambda^{\star}$ the spectral radius $r\left(T_{\lambda}\right)$ of $T_{\lambda}$ satisfies

$$
0<r\left(T_{\lambda}\right)<1 \text {. }
$$

Hence $B_{\lambda}=L_{\lambda}+\omega I-\left(M_{\lambda}+\omega I\right)=\left(\lambda^{-1} L_{1}+\omega I\right)\left(I-T_{\lambda}\right)$ is invertible with $B_{\lambda}^{-1}=\left(I-T_{\lambda}\right)^{-1} K_{\omega, \lambda}$. It holds that $B_{\lambda}^{-1}$ is positive and compact since $\left(I-T_{\lambda}\right)^{-1}$ is a positive bounded operator and $K_{\omega, \lambda}$ is a positive compact operator. Moreover, since $r\left(T_{\lambda}\right)<1$ it follows from [23, Lemma 1.4] that $B_{\lambda}^{-1}$ is irreducible, and hence the spectral radius $r_{\lambda}=r\left(B_{\lambda}^{-1}\right)$ is positive. By the Krein-Rutman Theorem $r_{\lambda}$ corresponds to a positive eigenfunction. 
Lemma 4.4. For all $\nu>0$ the operator $B_{\lambda}+\nu I$ is invertible. The inverse $\left(B_{\lambda}+\nu I\right)^{-1} \in \mathcal{L}(X)$ is positive and compact and its spectral radius is given by $r\left(\left(B_{\lambda}+\nu I\right)^{-1}\right)=\left(r_{\lambda}^{-1}+\nu\right)^{-1}$.

Proof. Let $k=\omega+\nu$. Then

$$
\begin{aligned}
B_{\lambda}+\nu I & =L_{\lambda}+k I-\left(M_{\lambda}+\omega I\right) \\
& =\left(L_{\lambda}+k I\right)\left(I-K_{k, \lambda}\left(M_{\lambda}+\omega I\right)\right) .
\end{aligned}
$$

The operator $K_{k, \lambda}\left(M_{\lambda}+\omega I\right)$ is also positive, compact and irreducible. Again using the Krein-Rutman Theorem we find that $r\left(K_{k, \lambda}\left(M_{\lambda}+\omega I\right)\right)$ is an eigenvalue of the adjoint operator $\left(K_{k, \lambda}\left(M_{\lambda}+\omega I\right)\right)^{*}$ pertaining to a positive functional, say $\Upsilon_{k, \lambda}$. Let $h_{\lambda}$ be the positive eigenfunction of $B_{\lambda}^{-1}$ corresponding to the eigenvalue $r_{\lambda}$ as in Lemma 4.3. It holds that

$$
h_{\lambda}=\left(r_{\lambda}^{-1}+k-\omega\right) K_{k, \lambda} h_{\lambda}+K_{k, \lambda}\left(M_{\lambda}+\omega I\right) h_{\lambda} .
$$

Using (42) it follows that

$$
\begin{aligned}
\left\langle h_{\lambda}, \Upsilon_{k, \lambda}\right\rangle & =\left(r_{\lambda}^{-1}+k-\omega\right)\left\langle K_{k, \lambda} h_{\lambda}, \Upsilon_{k, \lambda}\right\rangle+\left\langle K_{k, \lambda}\left(M_{\lambda}+\omega I\right) h_{\lambda}, \Upsilon_{k, \lambda}\right\rangle \\
& \geq\left\langle K_{k, \lambda}\left(M_{\lambda}+\omega I\right) h_{\lambda}, \Upsilon_{k, \lambda}\right\rangle \\
& =r\left(K_{k, \lambda}\left(M_{\lambda}+\omega I\right)\right)\left\langle h_{\lambda}, \Upsilon_{k, \lambda}\right\rangle .
\end{aligned}
$$

Hence $r\left(K_{k, \lambda}\left(M_{\lambda}+\omega I\right)\right)<1$ and the operator $B_{\lambda}+\nu I$ is invertible with

$$
\left(B_{\lambda}+\nu I\right)^{-1}=\left(I-K_{k, \lambda}\left(M_{\lambda}+\omega I\right)\right)^{-1}\left(L_{\lambda}+k I\right)^{-1} .
$$

Moreover $\left(B_{\lambda}+\nu I\right)^{-1}$ is compact and positive and irreducible. Since

$$
\left(B_{\lambda}+\nu I\right)^{-1} h_{\lambda}=\left(r_{\lambda}^{-1}+\nu\right)^{-1} h_{\lambda}
$$

we see that $\left(r_{\lambda}^{-1}+\nu\right)^{-1}$ is an eigenvalue to which a positive eigenfunction pertains. It then follows from the irreducibility of $\left(B_{\lambda}+\nu I\right)^{-1}$ that the spectral radius of this operator must be $\left(r_{\lambda}^{-1}+\nu\right)^{-1}$.

Lemma 4.5. If $\mu \in \mathbb{C}$ is such that $\operatorname{Re} \mu<r_{\lambda}^{-1}$ then $\mu$ is in the resolvent set of $B_{\lambda}$.

Proof. Let $h \in X$ be arbitrary and consider the equation

$$
B_{\lambda} g-\mu g=h,
$$

where $\operatorname{Re} \mu<r_{\lambda}^{-1}$. Choose $\nu \in \mathbb{R}$ large enough such that

$$
(\operatorname{Re} \mu)^{2}+2 \nu\left(\operatorname{Re} \mu-r_{\lambda}^{-1}\right)+(\operatorname{Im} \mu)^{2}<r_{\lambda}^{-2}
$$

and $\mu+\nu \neq 0$. Then

$$
\begin{aligned}
|\mu+\nu|^{2} & =(\operatorname{Re} \mu)^{2}+2 \nu \operatorname{Re} \mu+\nu^{2}+(\operatorname{Im} \mu)^{2} \\
& <r_{\lambda}^{-2}+2 \nu r_{\lambda}^{-1}+\nu^{2}
\end{aligned}
$$


and hence $0<|\mu+\nu|<r_{\lambda}^{-1}+\nu$. Equation (43) is equivalent with

$$
\left(B_{\lambda}+\nu I\right) g-(\mu+\nu) g=h .
$$

Using Lemma 4.4 we can rewrite this as

$$
\left(\left(B_{\lambda}+\nu I\right)^{-1}-(\mu+\nu)^{-1} I\right) g=-(\mu+\nu)^{-1}\left(B_{\lambda}+\nu I\right)^{-1} h .
$$

Since $\left|(\mu+\nu)^{-1}\right|>\left(r_{\lambda}^{-1}+\nu\right)^{-1}=r\left(\left(B_{\lambda}+\nu I\right)^{-1}\right)$ we have that $(\mu+\nu)^{-1}$ is in the resolvent set $\left(B_{\lambda}+\nu I\right)^{-1}$ and hence (44) has a unique solution. It follows from the closed graph theorem that $\mu$ is in the resolvent set of $B_{\lambda}$.

Proof of Theorem 2.2. Consider the operator $A_{\lambda}$ defined in (7). It holds that $\mu$ is in the resolvent set of $A_{\lambda}$ if and only if $\mu / \lambda$ is in the resolvent set of $B_{\lambda}$. Indeed if $\mu / \lambda$ is in the resolvent set of $B_{\lambda}$ the operator defined by

$$
(u, v) \mapsto(\varphi, \beta \varphi-\beta \psi)
$$

where $(\varphi, \psi):=\lambda^{-1}\left(B_{\lambda}-\mu / \lambda\right)^{-1}\left(u, u-\frac{1}{\beta} v\right)$ is directly seen to be $\left(A_{\lambda}-\mu\right)^{-1}$. Conversely, for $\mu$ in the resolvent set of $A_{\lambda}$ the operator defined by

$$
(u, w) \mapsto(\varphi, \varphi-(1 / \beta) \theta)
$$

where $(\varphi, \theta):=\lambda\left(A_{\lambda}-\mu I\right)^{-1}(u, \beta u-\beta w)$ is $\left(B_{\lambda}-\mu / \lambda\right)^{-1}$. Hence, using the last lemma we have that all $\mu \in \mathbb{C}$ with $\operatorname{Re} \mu \leq \nu_{\lambda}:=\lambda r_{\lambda}^{-1}$ that $\mu$ is in the resolvent set of $A_{\lambda}$.

Proof of Theorem 2.3. The theorem follows directly from Lemma 3.5 and the fact that $\Lambda(\lambda)$ is the unique solution in $\left[Z_{\lambda}, Y\right]$.

\section{A. Appendix.}

We recall some facts about quasimonotone systems. We remark that in this section $\Omega$ may be an unbounded domain.

Definition A.1. A system of elliptic equations

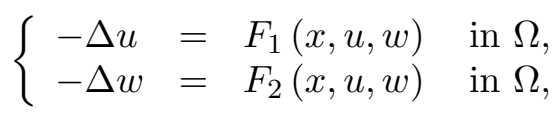

with $F_{i} \in C^{1}(\bar{\Omega} \times \mathbb{R} \times \mathbb{R})$ is called quasimonotone if $\frac{\partial F_{1}}{\partial u}(x, u, w) \geq 0 \quad$ and $\quad \frac{\partial F_{2}}{\partial w}(x, u, w) \geq 0$ for all $(x, u, w) \in \bar{\Omega} \times \mathbb{R} \times \mathbb{R}$.

This definition suffices for our purposes. For a more general definition we refer to [16]. 
Definition A.2. A pair $(u, w) \in C(\bar{\Omega}) \times C(\bar{\Omega})$ is called a subsolution to the problem

$$
\left\{\begin{aligned}
-\Delta u & =F_{1}(x, u, w) & & \text { in } \Omega, \\
-\Delta w & =F_{2}(x, u, w) & & \text { in } \Omega, \\
(u, w) & =(\varphi, \psi) & & \text { on } \Gamma=\partial \Omega
\end{aligned}\right.
$$

with $\varphi, \psi \in C(\Gamma)$ if

(1) it holds in $\mathcal{D}^{\prime}(\Omega)$-sense that

$$
\begin{aligned}
-\Delta u & \leq F_{1}(x, u, w), \\
-\Delta w & \leq F_{2}(x, u, w) ;
\end{aligned}
$$

(2) $(u, w) \leq(\varphi, \psi)$ on $\Gamma$.

Supersolutions are defined by reversing the inequality signs. If $(u, w)$ is both a subsolution and a supersolution then it is called a $C$-solution.

We note that if $\Omega$ is a bounded smooth domain and $F_{1}, F_{2}$ are $C^{1}$ then a $C$-solution $(u, w)$ is in $C^{2}(\bar{\Omega}) \times C^{2}(\bar{\Omega})$. We often use the following results from [16, Theorem 1.3].

Proposition A.3. Let $\Omega$ be a bounded smooth domain and assume that (46) is quasimonotone.

(1) If $\left(u_{i}, w_{i}\right), i=1,2$, are subsolutions to this system then $(u, w)$ defined by

$$
(u(x), w(x)):=\left(\max _{1,2}\left\{u_{i}(x)\right\}, \max _{1,2}\left\{w_{i}(x)\right\}\right)
$$

is again a subsolution to (46).

(2) If $(\underline{u}, \underline{w})$ is a subsolution and $(\bar{u}, \bar{w})$ a supersolution to (46) then there exists a $C$-solution $(u, w)$ to (46) with

$$
(\underline{u}, \underline{w}) \leq(u, w) \leq(\bar{u}, \bar{w}) .
$$

We give some results for $\Omega=\mathbb{R}_{+}^{N}:=\left\{\left(x_{1}, x^{\prime}\right) ; x_{1} \in \mathbb{R}, x^{\prime} \in \mathbb{R}^{N-1}\right\}$. The first is that one has also for quasimonotone systems the existence of a minimal and maximal solutions between an ordered pair of sub- and supersolutions.

Lemma A.4. Consider the following halfspace problem:

$$
\left\{\begin{aligned}
-\Delta u & =F_{1}(u, w) & & \text { in } \mathbb{R}_{+}^{N}, \\
-\Delta w & =F_{2}(u, w) & & \text { in } \mathbb{R}_{+}^{N}, \\
u & =w=0 & & \text { on } \partial \mathbb{R}_{+}^{N},
\end{aligned}\right.
$$

with $F_{i} \in C^{1, \alpha}(\mathbb{R} \times \mathbb{R}), 0<\alpha<1$, and suppose this system is quasimonotone. If there exists a bounded subsolution $(\underline{u}, \underline{w})$ and bounded supersolution $(\bar{u}, \bar{w})$ to this system with $(\underline{u}, \underline{w}) \leq(\bar{u}, \bar{w})$, then there exist a maximal and a minimal $C^{2, \alpha}$-solution in the order interval $[(\underline{u}, \underline{w}),(\bar{u}, \bar{w})]$ to this problem. 
The proof of this lemma is almost the same as for bounded domains. We only observe that if $\omega>0$ is such that $\frac{\partial}{\partial u} F_{1}(u, w)+\omega \geq 0$ and $\frac{\partial}{\partial w} F_{2}(u, w)+$ $\omega \geq 0$ for $(\underline{u}, \underline{w}) \leq(u, w) \leq(\bar{u}, \bar{w})$ then one can define inductively

$$
\left(u_{0}, w_{0}\right)=(\underline{u}, \underline{w}), \quad\left(u_{n+1}, w_{n+1}\right)=T\left(u_{n}, w_{n}\right), \quad n=0,1,2, \ldots
$$

with $(u, w)=T\left(u_{n}, w_{n}\right)$ the unique solution to the linear problem

$$
\left\{\begin{aligned}
\left(-\Delta+\omega_{1}\right) u & =F_{1}\left(u_{n}, w_{n}\right)+\omega u_{n} & & \text { in } \mathbb{R}_{+}^{N}, \\
\left(-\Delta+\omega_{2}\right) w & =F_{2}\left(u_{n}, w_{n}\right)+\omega w_{n} & & \text { in } \mathbb{R}_{+}^{N}, \\
u & =w=0 & & \text { on } \partial \mathbb{R}_{+}^{N} .
\end{aligned}\right.
$$

That this system has a unique solution follows from the fact that if $k>0$ and $g \in L^{\infty}\left(\mathbb{R}_{+}^{N}\right)$ then there exists a unique $u \in L^{\infty}\left(\mathbb{R}_{+}^{N}\right) \cap C\left(\mathbb{R}_{+}^{N}\right)$ such that $-\Delta u+k u=f$ in $\mathcal{D}^{\prime}\left(\mathbb{R}_{+}^{N}\right)$-sense and $u=0$ on $\partial \mathbb{R}_{+}^{N}$, see see e.g., $[6$, Proposition 27, p. 635]. Since the system is quasimonotone we have, see also [16], that

$$
(\underline{u}, \underline{w}) \leq\left(u_{n}, w_{n}\right) \leq\left(u_{n+1}, w_{n+1}\right) \leq(\bar{u}, \bar{w}) \text { for } n=0,1,2, \ldots .
$$

Letting $n \rightarrow \infty$ one obtains a solution.

The next lemma is used to reduce the study of equations on $\mathbb{R}_{+}^{N}$ to the study of inequalities on $\mathbb{R}^{+}$.

Lemma A.5. Suppose that $(U, W) \in C^{2}\left(\mathbb{R}_{+}^{N}\right) \cap C\left(\overline{\mathbb{R}_{+}^{N}}\right)$ is a bounded solution of

$$
\left\{\begin{aligned}
-\Delta U & =F_{1}\left(x_{1}, U, W\right) & & \text { in } \mathbb{R}_{+}^{N}, \\
-\Delta W & =F_{2}\left(x_{1}, U, W\right) & & \text { in } \mathbb{R}_{+}^{N}, \\
U & =W=0 & & \text { on } \partial \mathbb{R}_{+}^{N},
\end{aligned}\right.
$$

with $F_{i}\left(x_{1}, s, t\right) \in C^{1, \alpha}\left(\overline{\mathbb{R}_{+}^{3}}\right)$ and $0<\alpha<1$. Assume (48) is quasimonotone and that $\left|F_{i}\left(x_{1}, s, t\right)\right| \leq h(s, t)$ with $h$ a continuous function on $\mathbb{R}^{2}$. Define $(u, w)$ by

$$
(u, w)\left(x_{1}\right):=\left(\sup _{x^{\prime} \in \mathbb{R}^{N-1}} U\left(x_{1}, x\right), \sup _{x^{\prime} \in \mathbb{R}^{N-1}} W\left(x_{1}, x\right)\right) .
$$

It holds that $u, w \in C[0, \infty)$ with $u(0)=w(0)=0$ and in $\mathcal{D}^{\prime}\left(\mathbb{R}^{+}\right)$-sense that

$$
\begin{aligned}
& -u^{\prime \prime} \leq F_{1}\left(x_{1}, u, w\right) \\
& -w^{\prime \prime} \leq F_{2}\left(x_{1}, u, w\right) .
\end{aligned}
$$

Proof. Since $U$ and $W$ are bounded, $\Delta U$ and $\Delta W$ are also bounded. From this and the fact that $U=W=0$ on $\partial \mathbb{R}_{+}^{N}$ one obtains by standard regularity results that $U, W \in C^{2, \alpha}\left(\overline{\mathbb{R}_{+}^{N}}\right)$. In particular we have uniform bounds on the first order derivatives of $U$ and $W$. 
Let $\left\{q_{j} ; j=1,2, \ldots\right\}$ be a numbering of $\mathbb{Q}^{N-1}$ and define the functions $U_{j}$ and $W_{j}$ on $\mathbb{R}_{+}^{N}$ by

$$
\left(U_{j}, W_{j}\right)(x)=(U, W)\left(x+\left(0, q_{j}\right)\right) .
$$

For $k=1,2, \ldots$, we define $\left(S_{k}, T_{k}\right)$ on $\mathbb{R}_{+}^{N}$ by

$$
\left(S_{k}, T_{k}\right)(x)=\left(\sup _{1 \leq j \leq k} U_{j}(x), \sup _{1 \leq j \leq k} W_{j}(x)\right),
$$

and let $(S, T)(x):=\lim _{k \rightarrow \infty}\left(S_{k}, T_{k}\right)(x)$. It follows from the uniform continuity of $U$ and $W$ that $(S(x), T(x))=\left(u\left(x_{1}\right), w\left(x_{1}\right)\right)$.

Since the system is quasimonotone it follows from Proposition A.3 and induction that in $\mathcal{D}^{\prime}\left(\mathbb{R}_{+}^{N}\right)$-sense $-\Delta S_{k} \leq F_{1}\left(x_{1}, S_{k}, T_{k}\right) z$ for every $k=1,2, \ldots$ By dominated convergence it then follows that $-\Delta u \leq F_{1}\left(x_{1}, u, w\right)$ in $\mathcal{D}^{\prime}\left(\mathbb{R}_{+}^{N}\right)$-sense In particular if $z_{1} \in \mathcal{D}^{+}\left(\mathbb{R}_{+}\right)$we set $z:=z_{1} z_{2}$ with $z_{2} \in$ $\mathcal{D}^{+}\left(\mathbb{R}^{N-1}\right), z_{2} \neq 0$ one sees that this implies (49).

Since $F_{1}\left(x_{1}, u, w\right)$ is bounded there exists $M>0$ such that $F_{1}\left(x_{1}, u, w\right)-$ $2 M \leq 0$ on $\mathbb{R}^{+}$. Then $-\left(u+M x_{1}^{2}\right)^{\prime \prime} \leq 0$ in $\mathcal{D}^{\prime}\left(\mathbb{R}_{+}\right)$-sense. Hence $x_{1} \mapsto$ $u\left(x_{1}\right)+M x_{1}^{2}$ is convex and consequently continuous on $(0, \infty)$. Since $\frac{\partial}{\partial x_{1}} U$ is uniformly bounded and $U\left(0, x^{\prime}\right)=0$ for all $x^{\prime} \in \mathbb{R}^{N-1}$, it follows that $u$ is continuous in 0 with $u(0)=0$. The result for $w$ is obtained mutatis mutandis.

Finally we prove a direct analogue for a quasimonotone system of the sweeping principle for scalar equations in [15]. Suppose that $\Gamma=\Gamma_{1} \cup \Gamma_{2}$ with $\Gamma_{1}, \Gamma_{2} \in C^{2}$ and $\Gamma_{1} \cap \Gamma_{2}=\emptyset$. Here $\Gamma_{i}$ may be empty. Let $e \in C^{1}(\bar{\Omega})$ be such that $e(x)>0$ for $x \in \Omega \cup \Gamma_{1}$ and $e(x)=0, \frac{\partial e}{\partial n}(x)<0$ for $x \in \Gamma_{2}$ where $n$ is the outward normal and let $C_{e}(\bar{\Omega})$ be as in (38), see also [1].

Proposition A.6. Suppose that (46) is quasimonotone. If $(u, w)$ is a supersolution, and $\left\{\left(u_{t}, w_{t}\right) ; t \in[0,1]\right\}$ is a family of subsolutions such that

(1) $\left(u_{t}, w_{t}\right)<\left(g_{1}, g_{2}\right)$ on $\Gamma_{1}$ and $\left(u_{t}, w_{t}\right)=\left(g_{1}, g_{2}\right)$ on $\Gamma_{2}$ for all $t \in[0,1]$;

(2) $t \mapsto u_{t}-u_{0}$ and $t \mapsto w_{t}-w_{0}$ is continuous from $[0,1]$ into $C_{e}(\bar{\Omega})$;

(3) $\left(u_{0}, w_{0}\right) \leq(u, w)$ in $\bar{\Omega}$;

(4) $u_{t} \neq u$ and $w_{t} \neq w$ for all $t \in[0,1]$;

then there exists $r>0$ such that $(u, w)-\left(u_{t}, w_{t}\right)>($ re, re $)$ for all $t \in[0,1]$.

Proof. Let $S=\left\{t \in[0,1] ;\left(u_{t}, w_{t}\right) \leq(u, w)\right.$ in $\left.\Omega\right\}$. By assumption $0 \in S$. Since convergence in $C_{e}(\bar{\Omega})$ implies pointwise convergence it follows that $S$ is closed. Let $t_{0} \in S$. It holds with $\omega$ large enough in $\mathcal{D}^{\prime}(\Omega)$-sense that

$$
\begin{aligned}
-\Delta\left(u-u_{t_{0}}\right)+\omega\left(u-u_{t_{0}}\right) \geq & F_{1}(u, w)+\omega u-F_{1}\left(u_{t_{0}}, w_{t_{0}}\right)+\omega u_{t_{0}} \\
= & F_{1}(u, w)+\omega u-F_{1}\left(u_{t_{0}}, w\right)+\omega u_{t_{0}} \\
& +F_{1}\left(u_{t_{0}}, w\right)-F_{1}\left(u_{t_{0}}, w_{t_{0}}\right) \geq 0 .
\end{aligned}
$$

Since $u \neq u_{t_{0}}$ there exists $s^{\prime}>0$ such that $u-u_{t_{0}}>s^{\prime} e_{0}$ with $e_{0}$ a $C^{1}(\bar{\Omega})$ function with $e(x)>0$ for $x \in \Omega, e_{0}(x)=0$ and $\frac{\partial e_{0}}{\partial n}(x)<0$ for $x \in \Gamma$, 
see [3, Corollary p. 581]. Since $u(x)-u_{t_{0}}(x)>0$ for $x \in \Gamma_{1}$ and $\Gamma_{1}$ is compact, there exists $s_{1}>0$ such $u-u_{t_{0}} \geq s_{1} e$. In the same way there exists $s_{2}$ such that $w-w_{t_{0}} \geq s_{2} e$. By hypothesis 2 there exists $\delta>0$ such that $\left\|u_{t}-u_{t_{0}}\right\|_{e},\left\|w_{t}-w_{t_{0}}\right\|_{e}<s / 2$ for all $t \in[0,1]$ for which $\left|t-t_{0}\right|<\delta$. This implies that for all such $t$ we have that $u_{t}-u_{t_{0}} \leq \frac{s}{2} e$ and hence $u-u_{t}=u-u_{t_{0}}-\left(u_{t_{0}}-u_{t}\right) \geq \frac{s}{2} e$ and in the same way $w-w_{t} \geq \frac{s}{2} e$. Hence $S$ is open and we have that $S=[0,1]$. By the compactness of $[0,1]$ and by hypotheses (2) it follows that there exists $r>0$ such that $u-u_{t} \geq r e$ and $w-w_{t} \geq r e$.

Acknowledgment. We thank a referee for a number of very useful comments.

\section{References}

[1] H. Amann, Fixed point equations and nonlinear eigenvalue problems in ordered Banach spaces, SIAM Rev., 18 (1976), 620-709.

[2] S.B. Angenent, Uniqueness of the solution of a semilinear boundary value problem, Math. Ann., 272 (1985), 129-138.

[3] Ph. Clément and L.A. Peletier, Positive superharmonic solutions to semilinear elliptic eigenvalue problems, J. Math. Anal. Appl., 100 (1984), 561-582.

[4] $\mathrm{Ph}$. Clément and G. Sweers, Existence and multiplicity results for a semilinear elliptic eigenvalue problem, Ann. Scuola Norm. Sup. Pisa Cl. Sci., 14(4) (1987), 97-121.

[5] E.N. Dancer and J. Wei, On the profile of solutions with two sharp layers to a singularly perturbed semilinear Dirichlet problem, Proc. Roy. Soc. Edinburgh, Sect. A, 127 (1997), 691-701.

[6] R. Dautray and J.-L. Lions, Mathematical Analysis and Numerical Methods for Science and Technology, Vol. 1, Berlin-Heidelberg-New York, Springer, 1990.

[7] D.G. de Figueiredo and E. Mitidieri, A maximum principle for an elliptic system and applications to semilinear problems, SIAM J. Math. Anal., 17 (1986), 881-895.

[8] B. Gidas, W.M. Ni and L. Nirenberg, Symmetry and related properties via the maximum principle, Comm. Math. Phys., 68 (1979), 209-243.

[9] G.A. Klaasen and E. Mitidieri, Standing wave solutions of a system derived from the FitzHugh-Nagumo equations for nerve conduction, SIAM J. Math. Anal., 17 (1986), 74-83.

[10] G.A. Klaasen and W.C. Troy, Standing wave solutions of a system of reaction diffusion equations derived from the FitzHugh-Nagumo equations for nerve conduction, SIAM J. Appl. Math., 44 (1984), 96-110.

[11] N.V. Krylov, Lectures on elliptic and parabolic equations in Hölder spaces, AMS Graduate studies in mathematics, Providence, 1996.

[12] A.C. Lazer and P.C. McKenna, On steady state solutions of a system of reactiondiffusion equations from biology, Nonlinear Anal., 6 (1982), 523-530.

[13] P.-L. Lions, On the existence of positive solutions of semilinear elliptic equations, SIAM Review, 24 (1982), 441-467.

[14] G. Mancini and E. Mitidieri, Positive solutions of some coercive-anticoercive elliptic systems, Ann. Fac. Sci. Toulouse. VII, 3 (1986), 257-292. 
[15] A. McNabb, Strong comparison theorems for elliptic equations of second order, J. Math. Mech., 10 (1961), 431-440.

[16] E. Mitidieri and G. Sweers, Existence of a maximum solution for quasimonotone elliptic systems, Diff. and Int. Eqns., 7 (1994), 1495-1510.

[17] L. Nirenberg, Topics in Nonlinear Functional Analysis, Courant Inst. Math. Sci. Notes, New York, 1974.

[18] B. de Pagter, Irreducible compact operators, Math. Z., 129 (1986), 149-153.

[19] C.J. Reinecke and G. Sweers, A positive solution on $\mathbb{R}^{N}$ to a system of elliptic equations of FitzHugh-Nagumo type, J. Diff. Eqns., 153 (1999), 292-312.

[20] _ A boundary layer solution to a semilinear elliptic system of of FitzHughNagumo type, C. R. Acad. Sci. Paris Sér. I, 329 (1999), 27-32.

[21] F. Rothe, Global existence of branches of stationary solutions for a system of reactiondiffusion equations from biology, Nonlinear Anal. Theory, Meth. Appl., 5 (1981), 487-498.

[22] J. Serrin, Nonlinear equations of second order, A.M.S. Symp. in Partial Diff. Eq., Berkeley, August 1971.

[23] G. Sweers, Strong positivity in $C(\bar{\Omega})$ for elliptic systems, Math. Z., 209 (1992), 251271.

[24] W.C. Troy, Symmetry properties of elliptic equations, J. Diff. Eqns., 42 (1981), 400413.

Received February 15, 1999 and revised August 3, 1999.

Department of Mathematics

Delft University of Technology

2600 GA DELFT

The Netherlands

E-mail address: WSKCJR@puknet.puk.ac.za

Department of Mathematics

Delft University of Technology

2600 GA DELFT

THE NeTHERLANDS

E-mail address: sweers@twi.tudelft.nl 